\title{
Robot Communication Via Motion: A Study on Modalities for Robot-to-Human Communication in the Field
}

\author{
MICHAEL FULTON, CHELSEY EDGE, and JUNAED SATTAR, University of Minnesota
}

\begin{abstract}
In this article, we propose, implement, and evaluate a motion-based communication system for field robots: robots that operate in dynamic, unstructured, outdoor environments. We perform two pilot studies to guide our development of the system, then evaluate it alongside an audio communication system, an LCD display, and a system of blinking LEDs. We compare the usage of these four systems with three different robots from up to five different viewpoints of interaction in a large study administered via Amazon Mechanical Turk. We contribute in two ways to the development of a more robust form of field human-robot interaction, wherein robots can select the most appropriate communication vector for a given situation and context. First, we contribute a motion-based communication system for field robots along with three baseline systems against which to test it. Second, we present results from our development of this motion system, showing that it is easier to learn than a baseline blinking LED system, viable for use underwater, aerial, and terrestrial field robots, and less negatively affected by adverse viewpoints than other communication methods.
\end{abstract}

CCS Concepts: • Human-centered computing $\rightarrow$ Empirical studies in interaction design; • Computer systems organization $\rightarrow$ Robotics; External interfaces for robotics;

Additional Key Words and Phrases: Robotics, human-robot-interaction, field robotics, non-humanoid robots

ACM Reference format:

Michael Fulton, Chelsey Edge, and Junaed Sattar. 2022. Robot Communication Via Motion: A Study on Modalities for Robot-to-Human Communication in the Field. Trans. Hum.-Robot Interact. 11, 2, Article 15 (February 2022), 40 pages.

https://doi.org/10.1145/3495245

\section{INTRODUCTION}

Robots were first a fiction, then a rare sight, and now are increasingly a part of life in post-industrial countries. The entry of robots into our lives began in safe, sequestered environments, away from direct human contact in university labs and on factory floors. However, robots now fly above orchards and fields [45, 56], swim into industrial facilities [10, 36] and natural preserves [61], and move loads in shipping warehouses [62] around the world. In this age of increased robotic ubiquity, it is critical for robots to interact accurately, efficiently, and safely with humans sharing the same complicated, disorganized spaces. To enable this robust human-robot interaction (HRI),

M. Fulton was supported by National Science Foundation GRFP grant 00074041 (Fellow ID 2019279948).

C. Edge and J. Sattar were supported by National Science Foundation grant IIS 1845364.

Authors' address: M. Fulton, C. Edge, and J. Sattar, University of Minnesota, 100 Union Street SE, Minneapolis, Minnesota, USA, 55455; emails: \{fulto081, edge0037, junaed\}@umn.edu.

Permission to make digital or hard copies of all or part of this work for personal or classroom use is granted without fee provided that copies are not made or distributed for profit or commercial advantage and that copies bear this notice and the full citation on the first page. Copyrights for components of this work owned by others than ACM must be honored. Abstracting with credit is permitted. To copy otherwise, or republish, to post on servers or to redistribute to lists, requires prior specific permission and/or a fee. Request permissions from permissions@acm.org.

(c) 2022 Association for Computing Machinery.

2573-9522/2022/02-ART15 \$15.00

https://doi.org/10.1145/3495245 

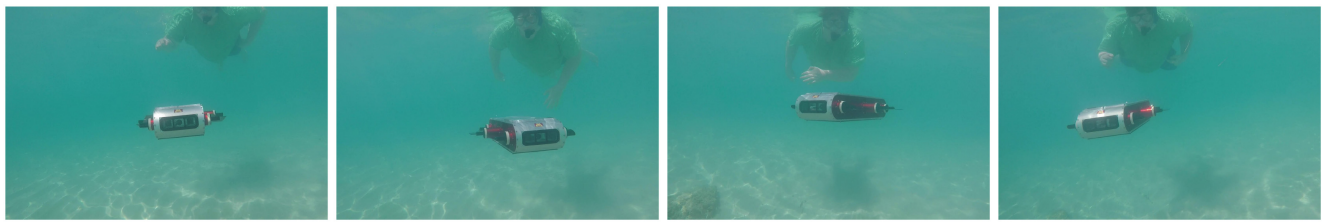

Fig. 1. The Aqua AUV in the Caribbean, indicating a "No" by shaking its "head" back and forth.

robots must have options on how to communicate with humans so that they can adapt to different situations, environments, and human collaborators. One commonly overlooked modality of communication, particularly for non-humanoid robots, is the motion of the robot itself. By employing the motion of its own body as a method of communication, nearly any robot could communicate with a human interactant without any other required hardware. To expand the communication method options for field robots of all kinds, we present in this work the design, development, early pilot studies, and in-depth testing of our motion-based communication system, Robot Communication Via Motion (RCVM).

Field environments are outdoor spaces which are not curated for robot operations, such as a field of crops, the surface of a lake, a forest, or the bottom of a river or ocean. These environments are irregular in their shape, disorganized in their layout, and dynamic in their state. They often come with their own challenges: degraded visual conditions, GPS and wireless signal attenuation, and strong winds or currents, to name a few. Robots tend to communicate in limited and highly structured ways, which become less effective in these challenging field environments. This brittle, failure-prone communication can lead to inefficiency, mission failure, and even endangerment of human collaborators. For example, many robots interact only via remote control systems, such as the controller/smartphone combinations used by many drones. Although remote controllers can pass a large amount of information both to and from the robot, they limit communication by forcing all interactions into a specific set of interface elements and by requiring human operators to carry a dedicated interaction device with them. The addition of this device and the attention it requires limits the interactant's ability to continue with other physical tasks and navigate the environment. HRI research has explored a variety of methods for natural robot-human communication without additional devices, such as spoken language [16, 52], facial expressions [26, 55], and displays built into the robot $[15,51]$. Even these other more natural communication modalities can be problematic in the field. Although speech-based communication works well indoors, outdoor audio can be difficult (perhaps even impossible) to decipher in extremely challenging circumstances, such as near a loud aerial robot or underwater, where audio is distorted. Additionally, not every robot has a shape that is conducive to mimicking human facial expressions or gestures, nor do they have the space for dedicated interaction hardware such as displays. This leaves a gap where field robots, due to their unique operating circumstances and task constrained designs, are left with highly structured and limited communication with human interactants via dedicated devices or with an inappropriate communication modality for the domain.

This gap first came to our attention in the context of underwater interactions, where the humanto-robot gestural language of our colleagues [33] was woefully outpacing the digital displays that our autonomous underwater vehicles (AUVs) used to respond to gestures. Our exploration of natural methods for robot-to-human communication underwater quickly brought us to a system of robot "body language gestures." This system, referred to as RCVM, uses "motions with meaning," called kinemes [24, 44], to communicate interaction phrases (short, self-contained ideas or phrases) as seen in Figure 1. RCVM has a phrasebook consisting of approximately 14 phrases but could easily be expanded far beyond this. To ensure that this system was worth exploring further, we 


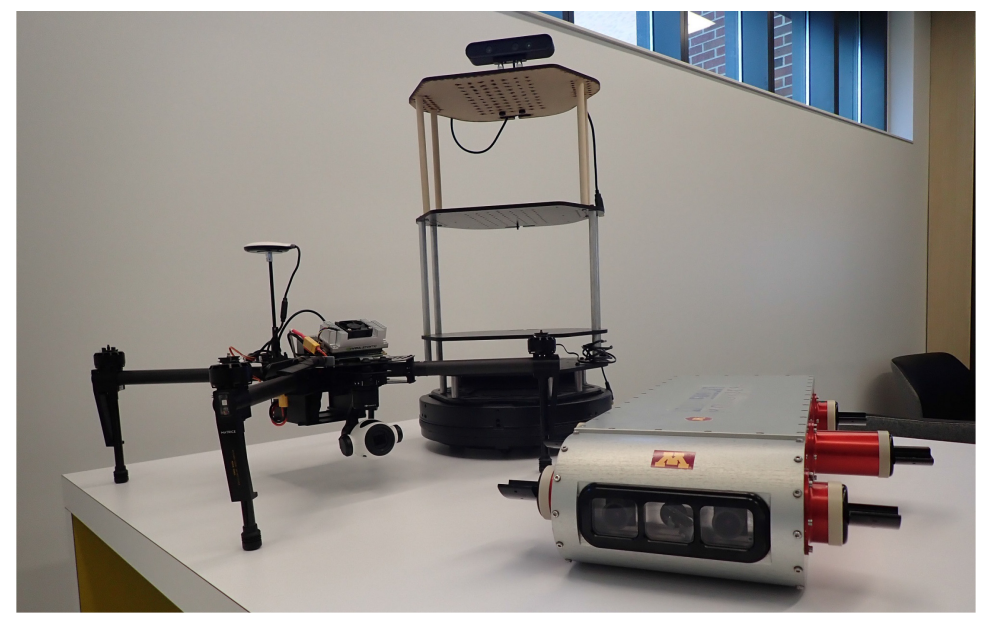

Fig. 2. The robots used in this work. From the left: Matrice 100 with Z3 Gimbal, Turtlebot 2, and Aqua.

tested a version of RCVM on a simulated AUV to determine how easily it could be learned and how well it performed compared to a communication system based on colored LED light codes (Pilot I). Following this, we began to test implementations of RCVM on multiple robots, exploring its capability to be used in aerial and terrestrial contexts (Pilot II). Finally, we completed our implementation of RCVM on three robots: a DJI Matrice 100 unmanned aerial vehicle (UAV) with a Zenmuse Z3 camera gimbal, the Aqua AUV [20], and a Turtlebot 2. Along with RCVM, we implemented three baseline systems utilizing colored LED light codes, digital text displays, and audible speech synthesis. We then tested the accuracy and efficiency of these systems (see Section 7.1), focusing on how each communication modality performed from various viewpoints, in different domains, and when communicating different types of information (multi-dimensional study). To do this, we performed a comparative study of our communication systems by recruiting participants using Amazon Mechanical Turk for an online survey administered through Qualtrics XM. This online study covered 355 participants from the United States, training each participant in the use of a communication system and then testing them on its use. The results from this study illuminate some quantitative differences between our four systems in terms of accuracy and efficiency of use, as well as some more subtle qualitative differences.

In this work, we make the following contributions:

- We propose and design a motion-based communication system to be used by field robots.

- We perform two pilot studies for this system, evaluating its usefulness first in simulation, then in a real-world implementation.

- We implement prototypes for this system and three baseline communication systems with which to compare our motion system across multiple robots.

- We perform a large comparative study on the accuracy and effectiveness of all these communication systems.

- We highlight the differences between the motion system and the comparison systems in terms of their efficiency and accuracy.

\section{RELATED WORK}

The primary subject of our research in this work is the use of motion as a communication modality for HRI in the field. Throughout this article, four modalities of robot-to-human communication 
are evaluated in different field environments across three studies. A motion-based system called $R C V M$-our primary target of investigation-appears in all three studies with LED code systems as a baseline in two studies (the first and third). LED code communication is joined by digital text display and audible speech synthesis as baseline modalities with which to compare RCVM in our final study. As such, we briefly present related work utilizing our four communication modalities: motion, lights, digital displays, and audible speech synthesis. Because our work is focused on field HRI, we will afford special focus on works that take place in field environments, and delineate underwater, aerial, and terrestrial works for each modality.

\subsection{Motion-Based Communication Modalities}

The use of motion as a vector of communication is an old concept, dating at least back to early attempts to create mechanical automatons mimicking the motion of humans and later research on creating androids and humanoid robots. Human communication emphasizes motions (conscious and unconscious) as modalities of communication, secondary only to spoken language. As such, it is natural for motion to be considered as an option for human-robot communication. However, the majority of motion-based communication has been explored in non-field environments, and for affective communication (communication of feelings) rather than transfer of information. Additionally, although motion can be used to communicate information from human to robot, the focus of our work is on the inverse problem, communicating information from robots to humans. For this reason, we will briefly discuss previous work on motion-based robot-to-human communication modalities, particularly as they relate to field robots and informative communication.

2.1.1 Underwater. Motion as a vector for communication underwater was largely unexplored until the publication of our study that comprises Pilot I in this work [24]. Underwater robots have typically relied on digital displays, purpose-built interaction devices, and lights for robot-tohuman communication, although some types of motion (e.g., diver following [32, 40, 48]) can be thought of as implicit communication. The primary way in which motion has been utilized for communication underwater is as a human-to-robot communication modality in hand gestures, as in the work of Islam et al. [33], or various contributors to the CADDY project such as in the work of Chavez et al. [15, 27]. However, in terms of explicit robot-to-human communication via motion, our previous work (included in this work as Pilot I) is the first investigation of this approach.

2.1.2 Aerial. In the field of aerial HRI, motion as a vector for communication has been widely explored. For the most part, research on the use of motion for UAV communication has taken the form of modifying flight paths to indicate direction or intent as in the work of Szafir et al. [53] and Duncan et al. [21]. These kinds of flight path "communicative overlays" aide human partners in working with these drones. Similar modifications to flight paths have been used by Cauchard et al. [14] to encode emotional states in drone flight. The other primary use of motion is in the use of human gestures $[38,42]$ or gaze and facial pose estimates $[28,41]$ of operators as control inputs. However, the work most closely related to ours is the Daedelus s-UAV (social UAV) [3], which used a motorized "head" with colored "eyes" to express emotions to interactants, capable of head tilting and nodding movements. This is very similar to our RCVM implementation for the Matrice UAV, which uses a motorized camera gimbal to mimic head motions. Both our RCVM implementation for the Matrice and the Daedelus s-UAV do not use flight path modification for communication, opting instead to use a single "head-like" appendage to achieve communication.

2.1.3 Terrestrial. By far, the largest amount of motion-based communication in previous work can be found in the terrestrial domain, in both field and non-field environments. Most motion-based communication works, however, use humanoid robots in their research, typically 
attempting to replicate human gestures on these platforms. Although fascinating, this type of research has little benefit for us, as nearly no field robots are humanoid in form. In terms of nonhumanoid motion interaction, Cindy Bethel's seminal thesis [6] is one of the most important works in the field, using the angle and motion of non-humanoid search and rescue robots to communicate affect from the robots to the humans they are helping. This work [6] was influential to ours as it introduced the idea of the variation in communication vector effectiveness at different distances (but not at different angles) and the concept of using multiple vectors of communication, either in concert or separately. Not having access to the same robots or communication systems, we are unable to reproduce Bethel's results directly (the content being communicated also differs), but our results on the effect of viewpoint on communication echo Bethel's work throughout. Bethel has studied and summarized non-facial, non-verbal affective communication quite extensively, providing useful surveys as well as her own contributions to the topic [7,8]. This type of motion-base affective communication has also been applied to zoomorphic robots such as a canine robot [39] and learned over time by an agent given the feedback of a human [50]. Non-affective motion communication has also been explored, from the use of a pan-tilt camera to simulate head nodding and other gestures [59] to using a digitally displayed virtual "head" to generate gaze cues to manage navigational conflicts with humans [29]. A large body of work has covered the topics of legible pointing [30], nonverbal communication for feedback in teaching by demonstration [31], the expressiveness of timing in manipulation motion [63], and the effect of different types of robot motion on human-robot collaboration outcomes [19] for terrestrial robots, largely in the realm of manipulators. These works all have some similarity to our study of RCVM, although we focus on informative communication (unlike Bethel [6]) via explicit motion gestures (unlike Dragan [18]), and use the base motion of a non-humanoid robot instead of adding human features (unlike Hart et al. [29]).

\subsection{Light-Based Communication Modalities}

Emitted light, either static or dynamic in its nature, is a common modality of communication used in robotics. In terms of non-verbal communication, light and motion are two of the most common spaces in which communication is done for HRI. Lights are likely used highly due to the importance placed on vision and color in human communications. Although the ability of humans to see and perceive color is varied, the majority of humans have non-color-deficient vision, influencing the way that communication is achieved around the world. Colored indicator lights are used to provide useful information of all types-even automobile traffic is controlled by colored light signals. It is no wonder, then, that HRI researchers so frequently turn to using light, both in terms of color and flashing patterns, to communicate information from robots to humans. We present a small selection of works that investigate the use of light as a robot-to-human communication modality, with an emphasis on field HRI.

2.2.1 Underwater. Emitted light is the only modality of communication that has been previously studied for underwater robot-to-human communication besides digital displays or the use of dedicated devices. Proposed by DeMarco et al. [17] in their influential case study of the effect of human-AUV communications on underwater work, it has typically been suggested that AUVs use their built-in lights (intended for illuminating environments for work) to communicate, by altering the brightness of the lights in a pattern. This proposal is quite reasonable, as divers already communicate with one another by using their flashlights to send signals to one another. We selected light codes as a baseline modality for Pilot I based on this fact and have continued to study it due to the widespread success it has found in other environments. Although our work is not focused 
on proposing light as a modality for underwater robot-to-human communication, it is one of the first to test this modality (particularly colored lights) in the underwater domain.

2.2.2 Aerial. Similar to motion, the use of lights as a method of communication has been explored much more extensively in aerial HRI than in underwater environments. Again, the work of Szafir et al. [54] is an important work in this space, showing that information can be communicated via lights. It is common for UAVs to have a small number of LED indicators built into their design, similar to the red and green navigation lights used on passenger airplanes. However, in their work, Szafir et al. [54] developed a system utilizing an LED strip wrapped around the entire UAV and used it to effectively indicate flight intent in a variety of ways (mimicking a turn signal, beacon, thruster, or human gaze cues). That work [54] influenced ours in its use of light sweeping (implying motion by turning lights on and off across a line). Without access to the same robots or communication systems, we were unable to reproduce the results of Szafir et al. [54] (the content being communicated also differs), but our findings about the ability of emitted light to communicate directional information concur with the results of Szafir et al. [54]. The work of Arroyo et al. [3] also utilizes lights as a communication vector, not for indicating intent but rather communicating emotional state in the form of colored "eyes."

2.2.3 Terrestrial. The world of terrestrial HRI has also used light as a form of communication for many years. The nonverbal communication work of Bethel [6] and Bethel and Murphy [8] utilizes illuminated color as a vector of communication, mostly for affective display. In terms of more information-focused communication, Baraka and Veloso [4] displayed information such as an intended path using an array of expressive lights around the robot's body. More recently, Hart et al. [29] used this same general concept as a baseline for their system of motion communication and found their gaze gestures to be more effective than expressive light arrays for resolving navigational conflicts.

\subsection{Digital Display Based Communication Modalities}

Digital displays of text are a very common communication modality for robots, with good reason. Text displays can contain detailed and dense information in only a few lines of text and can easily be used to display complex ideas that more abstract communication vectors such as motion or sound might not be able to. In the context of field robotics, digital displays pose something of a problem. They often do not achieve high fidelity in non-standard lighting conditions, such as high sunlight or slight haze, which are often encountered in field environments. They also only work within small viewing angles, impose weight and space constraints, and add to the electrical load of a robot. For these reasons, alghouth digital displays are used in field HRI, they are less interesting as an area of research. They have been used in underwater robots [27, 57, 58], aerial robots (either in separate, connected devices [5] or integrated into the robot [37]), and in myriad terrestrial robots [51]. Although digital displays have been used effectively in the field and are thus a fair baseline modality, they still struggle with the constraints of field environments and can likely be outperformed by other modalities.

\subsection{Speech Synthesis Based Communication Modalities}

Our last communication modality, audible speech synthesis, is another standby of HRI in non-field environments. The reasons for this are obvious: spoken language is the primary communication modality for the majority of humans around the world. Therefore, it is natural to use the closest analog to human speech for robot-to-human communication: synthesized speech. However, audio can be difficult to understand in field environments, particularly underwater, depending on the ambient noise and equipment worn by a diver. For this reason, synthetic speech has not been 
Table 1. Changes in Interaction Phrases, as Described in Section 3

\begin{tabular}{|c|c|c|c|c|c|c|}
\hline$\#$ & Interaction Phrase & Meaning & Pilot I Status & Pilot II Status & MTurk Study Status & Phrase Type \\
\hline 0 & Affirmative & Yes & Animated & Implemented & Implemented & Conversational \\
\hline 1 & Negative & No & Animated & Implemented & Implemented & Conversational \\
\hline 2 & Possible & Maybe & Animated & Eliminated & Eliminated & Conversational \\
\hline 3 & Ascend & Move up & Animated & Replaced with \#5 & Replaced with \#5 & Spatial \\
\hline 4 & Descend & Move down & Animated & Replaced with \#5 & Replaced with \#5 & Spatial \\
\hline 5 & Directional & Move in [direction] & Not created & Implemented & Implemented & Spatial \\
\hline 6 & Stay & Remain where you are & Animated & Implemented & Implemented & Spatial \\
\hline 7 & Attention & Look at me & Animated & Unused & Implemented & Conversational \\
\hline 8 & Danger & Danger nearby & Animated & Implemented & Implemented & Complex \\
\hline 9 & Follow Me & Follow me & Animated & Implemented & Implemented & Complex \\
\hline 10 & Malfunction & Something is wrong & Animated & Unused & Implemented & Complex \\
\hline 11 & Repeat Previous & Repeat last instruction & Animated & Implemented & Implemented & Conversational \\
\hline 12 & Object Indication & Object here...[direction] & Animated & Implemented & Implemented & Spatial \\
\hline 13 & Battery Indicator & Battery level is...[battery] & Animated & Implemented & Implemented & Complex \\
\hline 14 & Lost & The robot is lost & Animated & Unused & Implemented & Complex \\
\hline
\end{tabular}

Bold text indicates a modification.

applied in field environments nearly as frequently as in non-field HRI. Non-verbal sound has used as a vector of communication by Bethel and Murphy [8], Breazeal and Fitzpatrick [11, 12], and Scheef et al. [49], to name a few, but only Bethel and Murphy's work can be considered field HRI, as Breazeal and Fitzpatrick's and Scheef et al.'s works deal with social robots in non-field settings.

\section{RCVM: DESIGN AND IMPLEMENTATION}

RCVM is a novel system for motion-based information communication for field robots. It operates by assigning a motion gesture (kineme) to a piece of information that needs to be communicated to an interactant. This protocol was initially designed for the Aqua AUV but has been extended to two other robots: the Matrice $100 \mathrm{UAV}$ and the Turtlebot2. In this section, we expand on the interaction phrases used for RCVM, the design philosophy of RCVM, its implementation on three robots, and the comparison systems developed for our studies.

\subsection{Interaction Phrases}

The first phase in developing our motion-based communication system, RCVM, was to select a common library of phrases for communication. These phrases, as well as their development over time, can be seen in Table 1 . The library was initially created with underwater communication in mind, based on the authors' experiences operating underwater robots in the field. To develop the interaction phrases, the authors listed as many phrases or concepts as they could in response to the question "What information do you want your robot to be able to tell you?" Through an informal iterative coding process, these phrases were combined and distilled into their more raw conceptual forms. For instance, multiple answers such as "rough water ahead," "shark nearby," and "small, confined space ahead" were distilled into the concept of danger being nearby, leading to interaction phrase 8, Danger. Although most of the interaction phrases remained unchanged in their meaning throughout the study, phrases 3 and 4 (Ascend and Descend) were made to be more general by replacing them with phrase 5, Directional, which indicates movement in any direction in 3D space. Additionally, phrase 2, Possible, was removed after the first study, based on discussions with divers on the types of phrases that would be useful. Researchers interested in replicating or extending this work should adapt the library of phrases to reflect the needs of their problem domain, or possibly create an entirely new phrase library. The authors recommend taking a similar approach to the preceding but sourcing the initial responses to questions such as "What information do you want your robot to be able to tell you?" from domain experts and possible users of the robot system. 

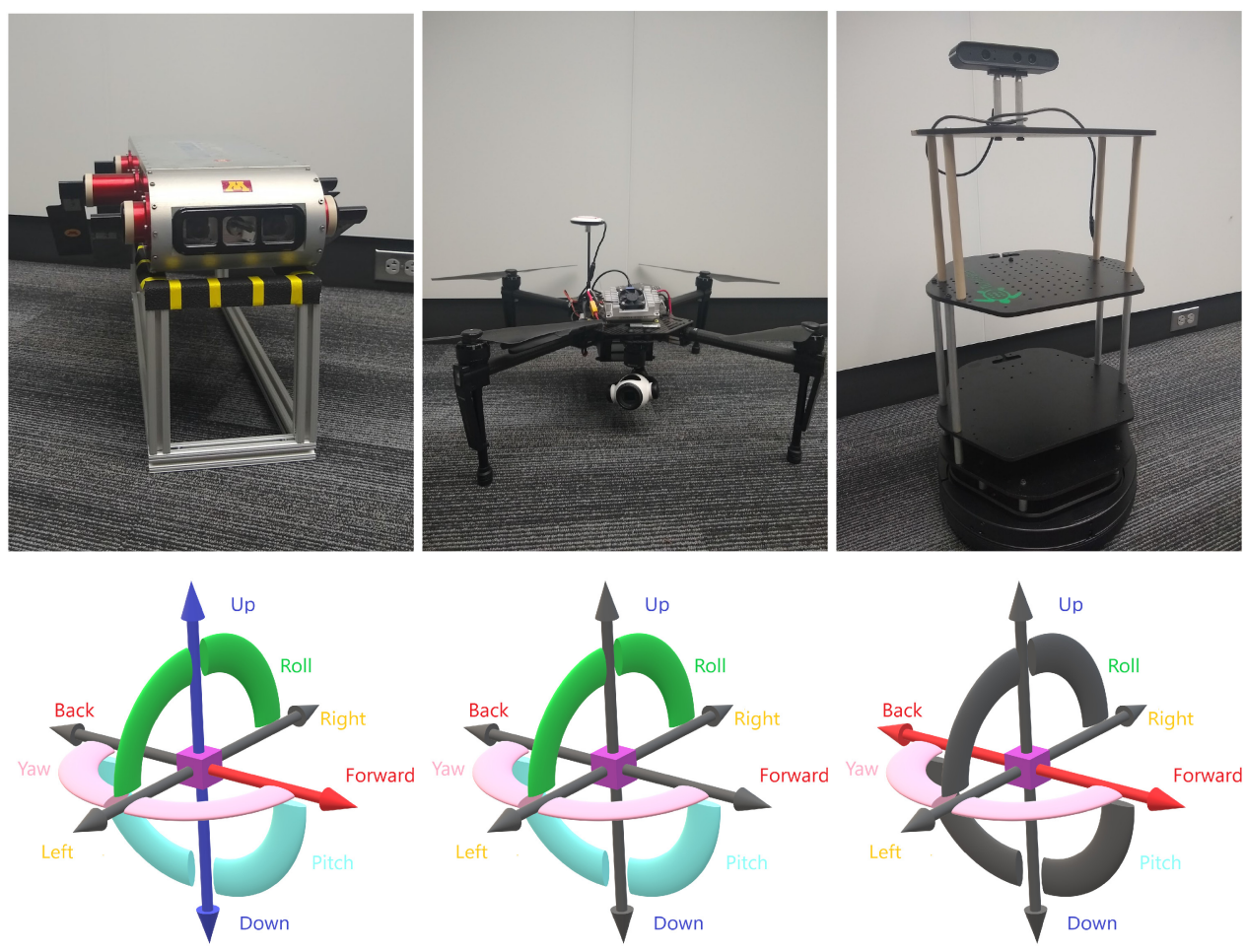

Fig. 3. The three robots used for our study of kineme communication and their motion capabilities. The robot in question cannot independently move on the grayed axes or rotation arcs, whereas colored axes and arcs are available for independent use. Note that the middle diagram shows the motion capabilities of the camera gimbal, not the Matrice 100.

Once those responses have been collected, iterative coding should be used to select the library of interaction phrases.

\subsection{RCVM Design}

As mentioned previously, RCVM operates by using a kineme (motion gesture) to represent a piece of information for an interactant. The word kineme comes from the Greek kinetikos, which means "of motion," and the suffix eme from phoneme (a unit of sound in spoken language), meaning some portion of linguistic structure. With the library of phrases to communicate selected as described in the previous section, the next task is to create a kineme for each interaction phrase. We looked to human body language for inspiration, emulating common human gestures whenever the shape and motion capabilities of the robot allowed it. For instance, in the case of the Matrice kinemes, the system was developed using a Z3 camera gimbal attached to the drone, which can easily mimic such head motions as nodding and shaking of the head (for Affirmative and Negative, respectively). Even when the robot's capabilities seemed less than friendly to human gesture emulation, it was still possible. For instance, the Aqua AUV has nothing remotely like a head that can be separately manipulated, but by yawing back and forth around the vertical axis (Figure 3), an approximation of a head shake can be achieved. When we could not copy human gestures directly, we sought to distill the interaction phrase we were communicating into directional information (e.g., turning toward an object that is meant to be indicated) or considered the associated emotions (e.g., fear 
for Danger, leading to the selection of quick, jerky motions). For all kinemes, we sought to keep their performance time relatively low so as to not interrupt other necessary motions the robot might need to make, such as station keeping or navigation to a new area. Although kinemes are intended to be used in periods of time dedicated only to communication, not during tasks, we wished to keep any interruption to task execution necessitated by kineme motion to a minimum. As the design of each interaction phrase's design is unique, we omit the detailed description of the design process here for the sake of brevity. For a full description of the design process for each kineme, as well as links to videos of each kineme on each robot, please view the appendix. Beyond the significant changes noted in Table 1, all kinemes were refined through the development process. Details about the specific implementation of the RCVM protocol for the three robots for which we have implemented it follow in the appropriate sections.

Note: Motion perception. As mentioned previously, in designing kinemes for RCVM, we attempt to emulate common human gestures whenever possible. This presents a question: what is a common gesture? The example provided, nodding and shaking of the head, might seem universal at first glance, depending on the reader's background. In fact, although head nodding is a widely recognized gesture meaning "Yes," there are some cultures that have different head gestures that may mean "Yes," or may apply different meanings to the head nod, even swapping the meaning to "No." For example, in some Balkan countries, including Bulgaria [1], the meaning of head nodding and shaking are reversed from the typical meanings. By this example, we can see that even the most seemingly universal gestures have cultural components to their perception. This effect obviously gets more pronounced as we move from head nodding and shaking to more complex (and therefore less common) gestures, such as hand gestures [2]. Although it is unlikely that anyone will be entirely lost on the meaning of a head nod, it is important for kineme designers to keep cultural components of the gestures they use as inspiration in mind as they transfer those gestures to a robot.

\subsection{RCVM Implementation}

To implement the kineme language for the three robots used in the following studies, ROS [47] nodes were written for each robot. Although these nodes differ significantly in the details of how they achieve their task, their overall construction is the same: the node responds to service calls by sending messages to the robot's motion control nodes that result in the appropriate kineme motion. The nodes were written to be compliant with ROS Kinetic or Melodic using Python 2.7. Robot-specific implementation details are explained in the following sections.

3.3.1 Aqua AUV. The Aqua vehicle is a 5-DOF underwater robot, mobilized by flippers. It is capable of independent forward, backward, upward, and downward motion, as well as rotation on all three axes. However, any motion taken can lead to drift, as there is significantly less friction acting on the robot than the friction stopping a terrestrial vehicle. This can lead to issues with the vehicle's motion control software, which is comprised of a Proportional-Integral-Derivative (PID) controller [43, Chapter 9.3] that attempts to move the robot to target angles and positions. If a water current pushes the robot in a direction, it may overcorrect and overshoot its target position, requiring another correction to reach the target and continue with the motion plan. Motions are also more likely to be somewhat different each time the kineme is completed, since the accuracy of the robot's motion control is not sufficient to exactly reproduce the same motion every time in the dynamic environment of the water. The Aqua family of robots are biomimetic in design, having a biologically inspired shape, with flippers that remind many observers of fish or cockroaches and two cameras in the front of its shell that are often referred to as eyes by onlookers. This serves it 
well in the development of kinemes, as kinemes based on human head gestures can be imitated with relative success.

3.3.2 Matrice 100 UAV. The Matrice 100 drone is an aerial vehicle capable of a variety of motions. However, for the development of kinemes on the vehicle, the motion being utilized is not that of the vehicle itself but rather of its camera, mounted on a Z3 gimbal. This gimbal is capable of a wide range of motion, with 320 degrees of pan, 120 degrees of tilt, and 120 degrees of roll. Because of its highly expressive motion, it is an excellent analog for the human head, making the implementation of many kinemes fairly easy. The gimbal also has very accurate control, making kinemes highly reproducible on this robot. A command sent to the gimbal control node will result in the target angle being reached quickly and accurately, with the primary point of failure being GPS errors. The gimbal considers its angles in relation to a GPS orientation, which, if it is disrupted, may cause the gimbal to misalign itself. This is not a problem in our use of the Z3 gimbal, as we provide the system with a simulated GPS fix, which remains constant. However, due to the robot's comparative size and the distances at which operators may be located, its effective "transmission range" may be limited.

3.3.3 Turtlebot 2. The Turtlebot 2 is perhaps the most limited of the platforms the kineme system was implemented on. Designed as a robot for teaching and use in office environments, the Turtlebot 2 is kinematically and dynamically most related to warehouse robots (operating on a differential drive that allows free movement on the $X$ axis and rotation around the $Z$ axis only). The Turtlebot was selected as an example of a terrestrial robot (not a field robot itself), as it was the most cost-effective platform available for development, and shares motion characteristics with actual terrestrial field robots ( $X$ axis translation and $Z$ axis rotation). To work within this limitation, certain features, such as wobbling after an abrupt stop, were employed to gain motion outside the 2D plane. The Turtlebot 2 robots used in these studies (seen in Figures 2 and 3) were modified to nearly double their original height by adding a second platform. This makes them larger and more unbalanced, leading to some interesting characteristics of their motion. The instability of the upper structure was used to telegraph a sense of fear in the Turtlebot's Danger kineme, and the greater height is useful for increasing visibility of the robot at a distance. This modification was not made for this study but provided an interesting example of a robot modified outside of its original specification (sensor rigs, manipulators, or other accessories added last minute) for mission purposes that was still capable of communicating information using motion.

\subsection{Comparison Systems}

To compare the capabilities of RCVM to other communication systems, we also developed three alternate systems: one based on flashing LEDs, one that uses text-to-speech audio, and one employing an LCD screen. These systems were designed using concepts observed in similar systems throughout field HRI works. Communication systems from previous works were not applied, since there are no established and accepted standard systems for use across domains. For instance, although many light-based communication systems exist, they all differ in important ways, and none could be generalized to all of our test platforms in a way that preserves their nature enough to compare previous results. Although our baseline systems do not represent the best possible application of their various communication modalities as they are not as fully integrated into the robots used, they provide a point of comparison for RCVM. It is expected that a purpose-built system utilizing the same modality that is closely integrated into the carrier robot and specialized for its domain would likely outperform these baseline systems. However, since such a theoretical specialized system would share the many of the properties as our baseline systems (the modality 
of communication-light, displayed words, or spoken words-to name just one), the baseline systems should provide an indication of how our motion communication system might perform in comparison to such a theoretical system. Links to videos of each interaction system performing all interaction phrases can be found in the appendix.

3.4.1 LED Communication System. The LED communication system was the first baseline system proposed to compare with the kineme system. Based on showing the user a series of colored lights flashing in a specified order and color, this system communicates the same ideas as all the other systems through light codes. Light is easily transmitted across distance and can survive a higher level of visual degradation than some other options, such as text-based communication on screens (see Section 3.4.3). Although the amount of information transmittable via colored lights is theoretically less than that allowed by motion, light and color are very natural human methods of information exchange. Think, for instance, of a red flashing light and the danger it indicates. There is, however, risk of confusion from those same intuitive meanings in light communication. Although a red light can mean danger, it can also indicate a powered-on state, Stop (as in a traffic signal), or a variety of other things. Nonetheless, the intuitive nature of colored lights, along with their relatively high resistance to poor visual conditions, made them a priority for comparing to RCVM.

The light code system was implemented using an Arduino to drive a series of LEDs. There were two versions of this system employed in the following studies: the 9-LED version (used in Pilot I, described in Section 4) and the 3-LED version (used in the MTurk Study described in Section 3.2). For the 9-LED version (Figure 4(b) and Table 2), three each of red, yellow, and green lights were arranged in a row. These LEDs were incapable of displaying other colors, so any mixing of colors was impossible. However, with the higher total number of lights, the possibilities for more complex patterns between the lights could be explored. In contrast, the 3-LED version employed only three RGB LEDs, arranged in a row. Because these LEDs were full RGB lights, they were capable of color mixing, displaying other colors beyond red, yellow, and green. Furthermore, directional light sweeping was used in the design of LED codes for the 3-LED version, following inspiration from Szafir et al. [54] and Baraka and Veloso [4] suggesting that this form of light communication could effectively demonstrate directional concepts. The sweeping takes the form of lights illuminating in sequence to indicate directional motion for the interaction phrases Object Left, Object Right, Move Left, and Move Right (differentiated by the color used). This feature, used for the LED codes displayed in the MTurk study, further improves the LED code system's capabilities. Although adapting LED systems to the specific robot being used and integrating them more fully would likely increase success, the 3-LED system used for the MTurk study is a modular, generalpurpose system that can easily be attached to all three robots used in our studies.

Note: Color perception. Color is highly useful for communicating emotional states and other ideas, because there are widespread connotations associated with different colors throughout most societies. However, care must be taken to consider societal perceptions of color when designing systems that utilize it. For instance, whereas red is most often associated with danger and love in Western countries, it is associated with luck and happiness in China. Although these large perception differences would likely not have effects unless a system reached wide distribution, smaller localized perception differences are possible and should be considered. In addition, a percentage of the population has color vision deficiency (commonly referred to as colorblindness). Care must be taken to ensure that systems utilizing color as an encoding space for communication consider the possible variations of color perception that exist, whether they arise from shared cultural ideas or physical traits of eye of the beholder. 
Table 2. [Pilot I] Kineme and LED Code Descriptions with Their Associated Meanings

\begin{tabular}{lll}
\hline Interaction Phrase & Kineme & LED Codes \\
\hline Affirmative & Head nod (pitch) & One solid green \\
Negative & Head shake (yaw) & One solid red \\
Possible & Head bobble (roll) & One solid yellow \\
Ascend & Ascend, look back, continue & Two solid yellow, one blinking green \\
Descend & Descend, look back, continue & Two solid yellow, one blinking green \\
Stay & Circle and barrel roll slowly & Two solid yellow, one blinking yellow \\
Attention & Roll heavily and erratically & Three quick blinking green \\
Danger & Look around then quick head shake & Three quick blinking red \\
Follow & Beckon with head, then swim away & Three blinking yellow \\
Malfunction & Slowly roll over & Three solid red \\
Repeat Previous & "Cock an ear" to the human & One blinking yellow light \\
Object Indication & Orient toward object, look at human & Two solid green, one yellow blinking \\
Battery Low & Small, slow loop-the-loop & One solid yellow, two blinking red \\
Battery Full & Large, fast loop-the-loop & One solid yellow, two blinking green \\
Lost & Look from side to side slowly & Three solid yellow \\
\hline
\end{tabular}

3.4.2 Audio Communication System. The audio communication system is comparatively straightforward: for each interaction phrase, a text-to-speech algorithm reads off a simple line of text specific to that phrase. For instance, for the Danger interaction phrase, the words "Danger nearby" are used. Although in some cases, such as underwater environments, audio is a tricky communication method, it is almost unparalleled in universal comprehension, for a very simple reason. For any given language, there are many people who can both read and understand the language when spoken. There are also a number of people who cannot read the language but can understand it when spoken. Additionally, although people's perception of colored lights and motion is likely influenced by their experiences with those communication methods (e.g., the types of warning and indicators lights they encounter in their daily life), words have a much more objective or agreed-upon meanings.

The audio system was implemented using a Bluetooth speaker, which played text-to-speech audio from the Google Cloud Text-to-Speech API. The use of the Google Cloud Text-to-Speech API could potentially have an effect on participant comprehension as compared to other TTS systems. The Google API was selected given its status as a consumer-facing state-of-the-art TTS system. The speaker used for transmission is temporarily mounted on each robot, pointing up or down, but not directly toward the interactant so that the audio communication should work equally well at any orientation to the robot. A speaker mounted facing toward the user would likely allow for higher distance communication at the cost of the omni-directionality of this system. This trade-off is a matter of choice. Another modification that could have allowed for higher distance communication is the increase of volume at different distances. However, the authors felt that audio should be kept at or below a level that would not be offensive to humans at "point-blank range," to avoid disruption of non-interactant humans, as well as any fauna in the area. This volume level was selected subjectively to the experimenter's experience and kept static for all experiments.

3.4.3 LCD Communication System. The LCD communication system works by displaying a short line of text on an LCD display for each interaction phrase. The text is often the same text used by the text-to-speech for audio, but it occasionally uses abbreviations to adapt to the smaller space. The display of written text has obvious advantages. Besides the more objective nature of word meanings described in Section 3.4.2, text on a display has the advantage of not requiring good audio transmission conditions (as underwater) and allowing multiple readings of the same 
text. These benefits are counterbalanced by the fact that this method is by far the most dependent on visual conditions and has the smallest viable viewing angle.

The display employed in our studies was a two-line, 16 characters-per-line backlit liquid crystal display, driven by an Arduino. We chose this size of display as being realistic for use in AUVs. Although some robots use much larger screens [57], in general, AUVs require small screens due to space and power budget restrictions, along with concerns about the ruggedness and rigidity of some types of displays. And although the types of displays used by UAVs and terrestrial robots were less considered in our selection of device, it should be noted that UAVs rarely carry digital displays of significant size due to weight restrictions, and terrestrial field robots are limited by the ruggedness requirements of their structures. Larger displays with bigger text would allow for text recognition at greater distance but would increase overhead and likelihood of damage to the display. The implementation of this system is very simple: for each interaction phrase, display the text on the LCD, beginning at the first character of the first line and splitting onto the second line if necessary. Once the text has been displayed, it is left on the display for 5 seconds, then cleared.

\section{PILOT STUDIES}

To develop and test our motion-based communication system, we conducted two pilot studies followed by periods of development. The results from these tests not only guided further development but also illuminated features of kineme communication that were not apparent from the beginning. First, we tested our kineme system by implementing them on a simulated version of the Aqua AUV and running a small study comparing the simulated system with the three-light version of the LED system. Next, we implemented kinemes for three robots and tested them through inperson tests with a full-loop communication study. This was a Wizard-of-Oz study, in which the autonomous communication ability of the robots was mimicked by a human operator triggering responses. In this section, we discuss the construction and results of these pilot studies, the full versions of which may be seen in their relevant papers (Fulton et al. [24] for Pilot I and Fulton et al. [23] for Pilot II).

\section{PILOT I: UNDERWATER KINEMES IN SIMULATION}

Our first pilot study on kineme communication employed a simulated underwater robot for a small, three-group study of 24 participants. The results of this study helped validate the concept of using motion as a vector for communication in field HRI and guided the further work described in later sections. When this study was conducted, the only application considered for kineme-based communication was that of underwater HRI, hence only an underwater robot was simulated (see Figure 4(a)). Since no previous work evaluating motion for communication underwater had been conducted, we chose to begin our work with a pilot study based on a simulated version of the robot to limit overhead costs. To have a point of comparison to work against, we tested not only our first, simulated version of kinemes for an underwater robot but also a baseline communication system based on colored LED codes (details in Section 3.4.1). The interaction phrases used in this study, along with descriptions of the kinemes and LED codes associated with them, can be found in Table 2. The structure of the study was as follows: users were randomly assigned one of three preparatory training levels (discussed further in Section 4.1.3), then were asked to test the kineme and LED code communication systems by watching randomly ordered videos of interaction phrases (kinemes or LED codes) and answering several questions. This study was submitted for determination to the University of Minnesota's Institutional Review Board and determined to be Not Human Research, making it exempt from full and continuing review. 


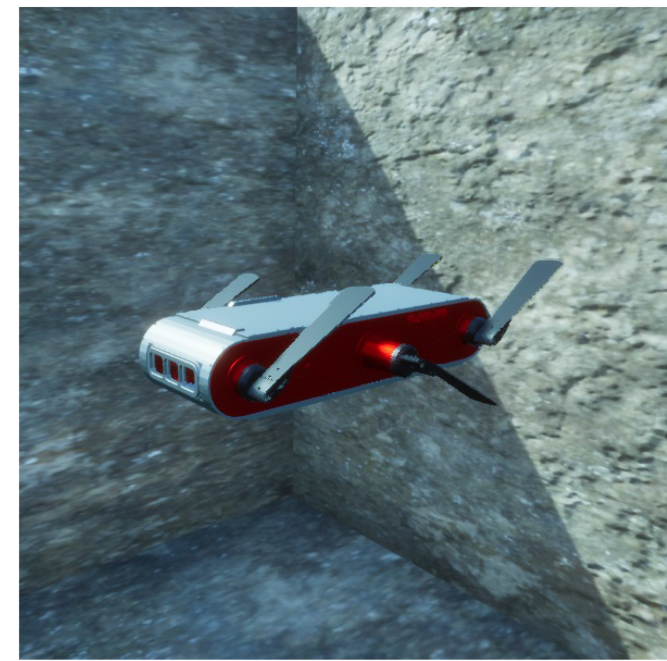

(a) Unreal Engine ${ }^{\mathrm{TM}}$ kineme implementation

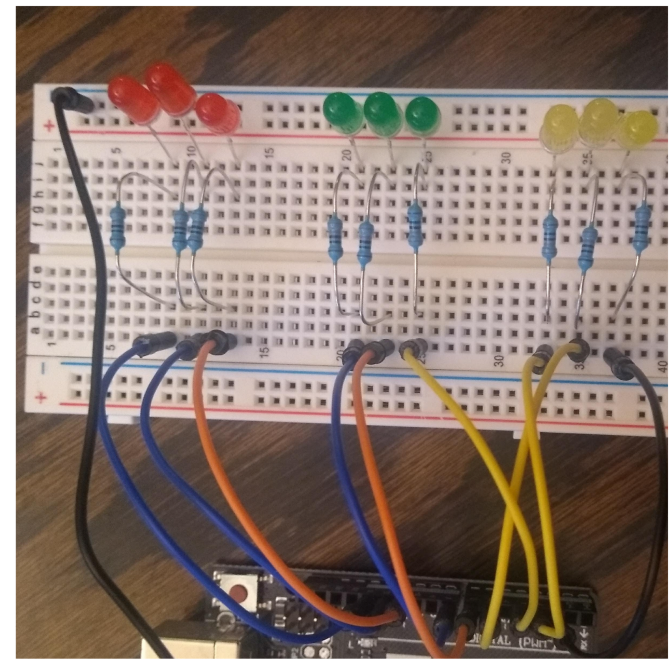

(b) Arduino ${ }^{\mathrm{TM}}$-based LED communication system.

Fig. 4. [Pilot I] Prototype communication systems used for the first pilot study.

\subsection{Experimental Design}

4.1.1 Hypotheses. The hypotheses we wished to test in this study were as follows:

- Kineme_Accuracy: Participants will interpret kinemes more accurately than LED codes.

- Kineme_Operational: Operational accuracy (accuracy for answers with a confidence $\geq 3$ on a scale from 1 to 5) for kinemes will be higher than kineme accuracy and higher than LED code operational accuracy.

- LED_Time: Time taken by participants to answer will be longer for kinemes than LED.

The analytical methods employed are described in Section 4.2, and the results are discussed in Section 4.4.

4.1.2 Population. The population $(\mathrm{N}=24)$ used in this study was mostly composed of students (16 male, 8 female) from the University of Minnesota-Twin Cities with a mean age of 22 years $(\sigma=3.3)$. To ensure that our population would be representative of non-expert users, participants were asked to rate their experience level with robots on a scale from 1 to 5 , with 1 being low $(\mu=1.54, \sigma=0.5)$. Although this group was limited in size and age range, the average level of robotics experience within the group was low. Because of this, although their experiences with the kineme system might generalize to the public at large, and is certainly useful for guiding the development of the system, further testing to verify a lack of effect from age or other factors would be beneficial. Participants were randomly put into one of three groups (eight members each), designated EDU0, EDU1, and EDU2 ordered by the amount of preparatory training they received for the communication task they were tested on.

4.1.3 Methods. Once they had been enrolled in the study and answered a demographic survey, each participant was provided with the same base information about the study: the fact that it was exploring underwater communication methods for robots. Based on the education group the participant was in, they were given the appropriate amount of training:

- EDU0: Participants were told which communication system they would be testing next (motion or LEDs). 
- EDU1: Participants were told the communication system, as well as a list of possible phrases.

- EDU2: Participants were told the communication system and shown videos of each kineme or LED code while being told the meaning.

Education was offered directly before testing each system and was not repeated upon participant request. Once a participant had been educated, they were shown videos of the kinemes or LED codes in random order. Additionally, the order of the system to be shown was randomized, further reducing the chances of order effects and producing more independent measures of each system and interaction phrase.

For each video, three pieces of information were recorded. First, the participant reported the meaning they perceived from the kineme or LED code in the form of a free answer. The method used to evaluate the correctness of each answer is discussed further in Section 4.2.1. The time, taken from the beginning of each video until the beginning of the answer, was recorded, along with the participant's confidence in their answer rated on a scale from 1 to 5 .

\subsection{Analysis}

4.2.1 Answer Rating Methods. In the study, each participant provided their answer to the question "What is the robot trying to say in this video" as a free answer, not restricted to a number of words or a specific set of choices. To determine correctness, a rubric of five categories the answers could fall into was defined:

(1) Entirely wrong

(2) Slight conceptual relation

(3) Partially correct

(4) Correct understanding, but worded differently

(5) Verbatim correct.

An earlier version of this study's analysis presented in the work of Fulton et al. [24] used a single expert rater following this scale. This creates a threat to internal validity from bias in the scoring of the participants' responses, so three additional raters with no other association with the study were asked to independently score each response. The inter-rater reliability measure of Krippendorff's alpha coefficient [34] is used to determine the level of reliability in the scoring of the raters. Krippendorf's alpha coefficient may be seen as a measurement of how the rater's agreement compares to the agreement of random selections, with $\alpha=1$ indicating far greater agreement than random (perfect reliability), $\alpha=0$ indicating the agreement expected of random (absence of reliability), and $\alpha<0$ indicating a greater amount of disagreement than expected of random selection (systemic disagreement). The three new raters' scores were found to have $\alpha=0.9137$, indicating strong evidence of reliability. When combined with the original expert rater, the statistic is $\alpha=0.9059$, still well within acceptable ranges. Therefore, the correctness scores of all four raters were averaged without weighting and used for all further analysis.

4.2.2 Hypothesis Testing. The kineme and light system are compared on the basis of the following criteria:

- Accuracy: The accuracy of a participant's understanding of a kineme or LED code, rated from 1 to 5 in order of increasing accuracy.

- Confidence: The confidence a participant has in their understanding of a kineme or LED code, rated from 1 to 5 in order of increasing confidence. 


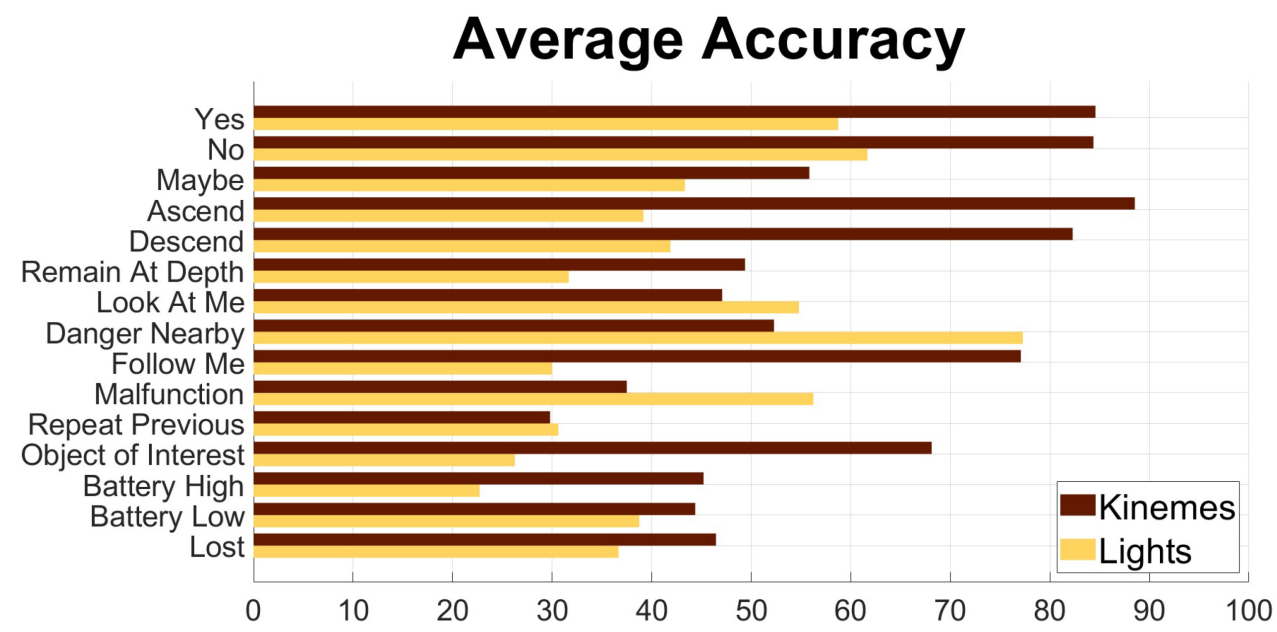

(a)

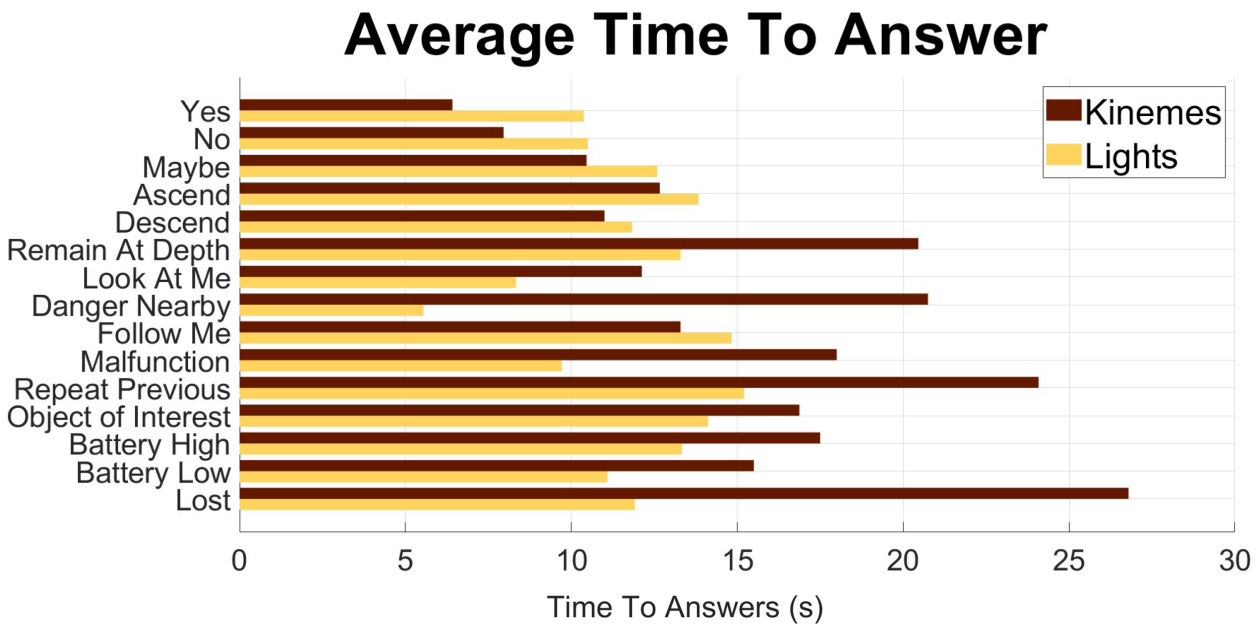

(b)

Fig. 5. [Pilot I] Results of the accuracy (a) and time to answer (b) for each interaction phrase, compared between the kineme and LED code systems. The kineme system typically achieves higher accuracy but has longer answer times than the LED code system.

- Operational accuracy: The same metric as accuracy but only taking answers rated at a confidence level of 3 or higher, representing the answers that participants would be likely to act on.

- Time to answer: The time it takes a participant to give the meaning of a kineme or LED code, measured in seconds from the beginning of the signal to the beginning of their answer.

Results for each kineme and LED code in terms of accuracy, operational accuracy, and time to answer can be found in Figure 5. These results show some kinemes that work better than their related LED codes and vice versa. To evaluate the hypotheses outlined in Section 4.1.1, we use a Mann-Whitney [35] test with Bonferroni correction [9]. The Mann-Whitney test is ideal for 


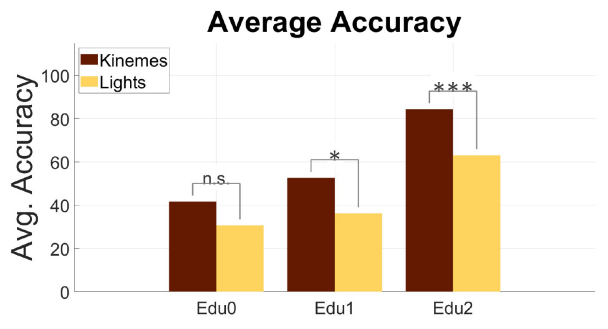

(a)

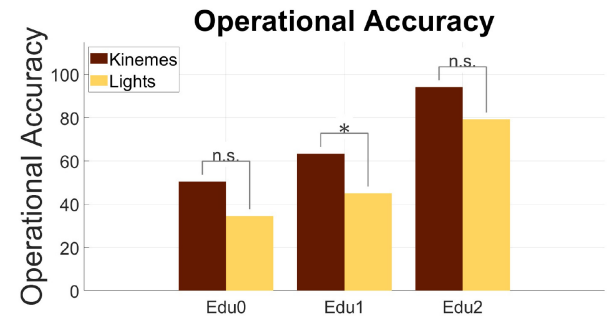

(b)

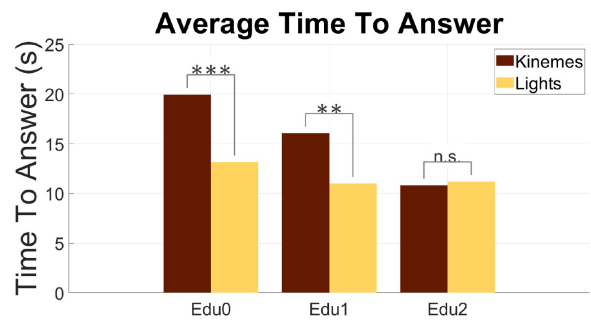

(c)

Fig. 6. [Pilot I] Hypothesis testing for Kineme Accuracy (a) Kineme_Operational (b), and LED_Time (c) Significance is represented with asterisks: ${ }^{*}(\alpha=0.05),{ }^{* *}(\alpha=0.01)$, and ${ }^{* * *}(\alpha=0.001)$. The kineme system achieves higher accuracy than the LED system with statistical significance at higher education levels and achieves an approximately equal average time to answer with the LED system at EDU2.

measuring the statistical effects of using the different systems in our trials, as it does not require normally distributed data. As we are conducting repeated simultaneous statistical tests, we employ Bonferroni correction (dividing the $\alpha$ for each test by the number of tests employed) to our MannWhitney hypothesis testing. Our overall significance level is $\alpha=0.05$.

\subsection{Results}

4.3.1 Statistical Results. In this section, we present the results of the preceding testing on the hypotheses outlined in Section 4.1.1 and can be found in Figure 6.

Kineme_Accuracy. We find that participants perceive kinemes more accurately at all education levels as seen in Figure 6(a). We additionally find statistically significant differences between systems at $\operatorname{EDU} 1(p=0.002, z=2.774)$ and $\operatorname{EDU} 2(p<0.001, z=3.824)$, but not EDU0. This implies that although kinemes may not be accurately understood if they were being viewed with no prior training or context, they are more accurately understood by users with a small amount of either. Therefore, we find that our hypothesis Kineme_Accuracy is supported for higher education levels but not statistically supported for situations in which participants have no training for the systems.

Kineme_Operational. Similar to the results of Kineme_Accuracy, we find that kinemes have greater operational accuracy at all education levels. However, the only statistically significant result is found at EDU1 ( $p=0.001, z=3.0716)$. This is unsurprising, as the confidence requirements of operational accuracy confine the answers used in this analysis to only the best. Nonetheless, kinemes retain a superiority to LEDs at all education levels (seen in Figure 6(b)), supporting the hypothesis with statistically significant support at EDU1. 
LED_Time. Last, in the case of LED_Time, we find statistically significant support for the hypothesis at EDU0 ( $p<0.001, z=-5.031)$ and EDU1 $(p<0.001, z=-3.398)$, but not at EDU2. Simply looking at the values, we can see that understanding kinemes takes an average of almost 10 seconds longer than LED codes at EDU0, 5 seconds longer at EDU1, and is approximately even at EDU2. This can be seen in Figure 6(c). This gives support for our hypothesis that LEDs will take less time to understand, with the exception that this does not hold true at high levels of education.

4.3.2 Participant Opinion. After completing their study, participants were given an exit survey, in which they were asked to rate the kineme and LED systems on a scale from 1 to 10 for several metrics. Participants rated kinemes easier to understand $(\mu=5.6, \sigma=2.2)$ than LEDs $(\mu=3.5, \sigma=$ 3.3). They also considered kinemes easier to learn $(\mu=7, \sigma=2.1)$ than LEDs $(\mu=5.5, \sigma=3.5)$. When asked, $71.4 \%$ of participants also preferred the kineme system overall and $66.7 \%$ felt it would be most effective from a significant distance. Last, when asked whether kinemes, LED codes, or an LCD screen (not analyzed in this study) would be best for an underwater communication system, $45.8 \%$ preferred the kineme system, compared to $37.5 \%$ for LEDs and $16.7 \%$ for the LCD.

\subsection{Discussion}

The performance of kinemes in this study, competitive with and even outperforming the LED code system (which is similar to previously used underwater communication systems), showed that kinemes could work well enough for underwater communication to be worth further investigation. Although the study employed simulated data and a small sample size, clear benefits to the kineme system could be seen. Participant accuracy for kineme identification is greater than for LED code identification overall, at every education level, with variation in this trend for individual kinemes. For instance, kinemes that mimicked human gestures toward directions (Ascend, Descend, Follow $\mathrm{Me}$ ) outperformed their equivalent LED codes, whereas LED codes that matched common uses of light (e.g., red lights for Danger or Malfunction) outperformed the kinemes. These differences in success based on the type of information being communicated formed the basis for our later multidimensional study, presented in Section 6. The results of this study formed a strong groundwork to build on and provided some initial understanding of the complex factors that comprise human perception of motion as meaning. Although the results did not establish a clear superiority of kinemes over LED codes, kinemes performed as well or better than LED codes in most instances. This legitimized the approach for further study with a physical implementation on robots.

\section{PILOT II: MULTI-PLATFORM IMPLEMENTATION}

In this section, we describe our second pilot: a small study using our first physical implementation of kinemes for three robots, evaluated on a small group of eight participants. The results of this pilot study helped lay the groundwork for our multi-dimensional study by revealing some initial qualities of physically implemented kinemes. Although this study was not large enough to draw strong conclusions about kineme effectiveness, it was the first test of an implemented kineme system on a physical robot, placed in the context of mock interaction sequences. It provided further validation that kinemes could be feasible in the real world, and showed that they worked on other platforms besides the Aqua robot, and in different environments. A group of eight participants was asked to interact with all three robots in a semi-randomized order, using a four-gesture language. The robot's responses would then suggest a course of action (unique to each kineme) that the participants could then take. This study was submitted for determination to the University of Minnesota's Institutional Review Board and determined to be Not Human Research, making it exempt from full and continuing review. 


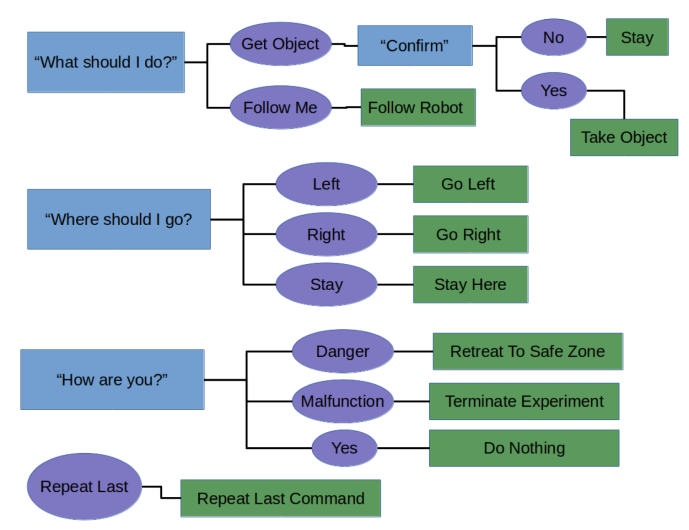

(a) Flow chart depicting the interaction process.

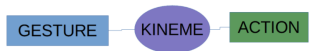

(b) Key to the above flowchart: Boxes are gestures (blue) or actions (green) of the human, while the purple ovals are kinemes generated by the robot.

Fig. 7. [Pilot II] How users interact with the robot in this study: a cycle of gestures, kinemes, and actions.

\subsection{Experimental Design}

5.1.1 Research Questions. In this study, we intended to explore several research questions, outlined in the following; this study is not hypothesis driven due to its small sample size but seeks to explore the following questions more qualitatively:

RQ1: Will kinemes remain reasonably accurate when implemented on a physical robot rather than a simulation?

RQ2: Will there be any perceptible differences in kineme success between different robots?

RQ3: Will kinemes work when placed into a full-loop communication system involving input and actions from the human interactant?

Due to the challenges of motion planning in each of the chosen robots' domains, it was important to test these questions with the physical robots, even on a small scale. This study provided confirmation that the results of our previous work were not successful merely due to the ease of control afforded by simulation, and it also offered interesting insights into the weak points of kineme implementations at this stage.

5.1.2 Population. The study population $(\mathrm{N}=8)$ was comprised of participants who were mostly 21 to 34 years old, $75 \%$ male and $25 \%$ female, and $55.66 \%$ Asian and $44.44 \%$ White or Caucasian. Participants self-reported their experience with robots on an ordinal scale from 0 to 100 , rating themselves at an average 49.20 out of 100 for marine robots, 54.29 for aerial robots, and 54.00 for terrestrial robots.

5.1.3 Methods. Prior to beginning the study, all participants were provided with educational material to be completed at their own pace. The educational material familiarized them with the study layout and flow (using the image in Figure 7), the gestures they would use for input, and the kinemes (shown in random order) for all three robots (also in random order). This preparatory training is very similar to the training received at EDU2 in Pilot I (described in Section 4.1.3). The 
primary difference is that in this study, participants were learning an entire interaction loop: their input options, the output options from the robot, and expected interaction flow. Participants took an average of 18 minutes to complete their education ( $\max =33$ minutes, $\min =9$ minutes) and completed the education an average of 15 hours before their first sessions with the robots ( $\max =$ 52 hours, $\min =1$ hour). Users were shown Figure 7 briefly before their first interaction session to refresh their memory.

After completing their education, participants were able to test the kinemes of each robot in a series of mock interaction sequences taking place either in lab (Matrice and Turtlebot2) or pool (Aqua) environments. Participants were told to ask the robot one of three questions, pay attention to the robot's response delivered via kineme, and take the appropriate action. A flow chart that shows the possible sequence of questions, kineme response, and actions can be found in Figure 7.

The question was communicated using a set of gestures based on relevant American Sign Language signs for "What should I do?," "Where should I go," and "How are you," with another sign for "Confirm." The users were told that the robot would observe their gestures and automatically select a kineme to display. In actuality, however, the kinemes were selected randomly by study staff in secret, with consideration given to displaying each kineme close to the same number of times across the study. Once they watched the selected kineme, the user then took the appropriate action based on their understanding of what the robot was trying to say to them.

After each interaction sequence, users were asked for a confidence score between 1 and 5 (5 being high) on the accuracy of the whole interaction. This confidence was recorded along with the total time of the interaction and the sequence of question to kineme to action. For each robot, participants completed an interaction session of between 10 and 15 interactions. Due to scheduling constraints, most participants were required to perform Turtlebot2 and Matrice robot interactions together (in random order), followed by the Aqua interactions soon after at the University of Minnesota Aquatic Center. For the Turtlebot2 and Matrice interactions, participants were standing in laboratory environments, approximately $1.5 \mathrm{~m}$ from the robot in question. For the Aqua interactions, participants swam in the pool with Aqua, approximately $1.5 \mathrm{~m}$ away from the robot. They surfaced as often as required for air, diving underwater to perform their hand gestures and observe Aqua's kineme responses. Last, after completion of each robot session, each participant was asked to fill out a survey with a variety of questions on their perception of the kinemes on each robot These questions included rating each kineme system on a 10-point scale for ease of understanding, speed of understanding, and how confusing they were, as well as questions about their perceived source of any mistakes they made.

\subsection{Results}

No hypothesis testing was done for this study since there were no independent variables besides robot choice in its design, and our sample size was rather small. Rather, we explore the results in a more qualitative way by observing the strengths and weaknesses of kinemes on different platforms through average accuracy, interaction time and confidence, and the measured confusion between kinemes.

5.2.1 Accuracy. The accuracy of the kineme systems as seen in Table 3 is lower than the accuracy for the Aqua kinemes in simulation as tested in Pilot I. The fact that accuracy is in the $60 \%$ to $70 \%$ realm is promising, as users are recognizing the kinemes at a rate far higher than random chance. The accuracy of the Matrice system is significantly higher than that of the other systems, possibly due to its similarities to a head or eye. Several participants remarked that it looked like a big eye, drawing comparisons to characters from popular media with similar 
Table 3. [Pilot II] Comparison between the Simulated Aqua from Pilot I and the Three Robot Kineme Systems from the Study

\begin{tabular}{lccc}
\hline System & Accuracy & Avg. Time & Avg. Conf. \\
\hline Aqua (Unreal) & $84.33 \%$ & 15.59 & 3.04 \\
Aqua & $60.00 \%$ & 34.75 & 3.01 \\
Matrice & $\mathbf{7 6 . 6 2 \%}$ & 24.41 & 3.76 \\
Turtlebot2 & $68.83 \%$ & $\mathbf{2 3 . 4}$ & $\mathbf{3 . 7 4}$ \\
\hline
\end{tabular}

All three robot kineme systems achieve reasonable accuracy levels, but there is a drop in accuracy when moving from the simulated Aqua to the physical one.

appearances (GLaDOS, Luxo Jr., etc.). The Turtlebot2 system comes second, with many participants remarking on how "cute" it was, indicating a high level of anthropomorphism. This is somewhat surprising, as the Turtlebot2's motion is the most constrained of the three robots and has the least anthropomorphic shape.

The ranking of the Aqua AUV as last is likely a result of several experiment-day malfunctions. For instance, a previously successful kineme, Follow Me, began to experience malfunctions in this study. The differences in inertia and friction between pool operation and simulation caused the "beckoning" motion of the kineme to look incorrect and the robot to not complete its turn. The kineme achieved only $33 \%$ accuracy, far below the accuracy seen in simulation. Additionally, using the kinemes in the pool is inherently more stressful than recognizing kinemes in a video in the lab, as the participant must remain focused enough to keep their footing when standing, consider the remaining air in their lungs while underwater, and so on. In addition, since participants were using a full-loop communication system involving gesture control, there were added cognitive loads related to recalling the gestures and remembering which actions were appropriate to take after identifying a kineme.

Average accuracy, response time, and confidence for each kineme across all three robots are summarized in Table 4. These results may also be seen in the confusion matrix shown in Table 5 . The meaning of most kinemes is being well identified by participants, with a few clear aberrations. The Indicate Movement and Indicate Object kinemes are not frequently confused with one another, which is a positive result, given that both are directionally focused. However, the Indicate Object kinemes for the Matrice, which contain a nod in the direction of an object, have been misidentified several times as the Affirmative kineme. Based on this result, we suggest that using one kineme in the composition of another may not be optimal. This was an instance where although we attempted to reduce overlap between kinemes wherever possible, some confusion appears to have happened. Further investigation on how to avoid confusion between kinemes with similar motions and how to design kinemes with distinct motions is warranted to explore this issue.

5.2.2 Confidence and Efficiency. In terms of average confidences, users have the most confidence in the Turtlebot2 interactions and the least in Aqua interactions. However, all systems achieve average confidence of 3 or higher, which is the halfway mark on the scale that users were given. The Affirmative, Indicate Stay, Negative, and Indicate Movement (Right) kinemes achieve the highest overall average confidences (all above 4). The Repeat Last kineme is the only kineme with average confidence below 3 , reducing the total average.

The average interaction time for all kineme systems is around 27 seconds, with the Turtlebot2 achieving the fastest times at 23.4, and the Aqua AUV the slowest at 34.75 seconds. These interaction times are affected by the duration of the kinemes in question, but the ease with which participants recognize each kineme also affects the interaction times. 
Table 4. [Pilot II] Accuracy, Interaction Time, and Confidence Per Kineme, Averaged Across All Robots

\begin{tabular}{lccc}
\hline Kineme & Accuracy & Avg. Time & Avg. Conf \\
\hline Affirmative & $\mathbf{9 5 . 0 0 \%}$ & 21.25 & $\mathbf{4 . 5 0}$ \\
Negative & $80.00 \%$ & $\mathbf{2 0 . 8 5}$ & 4.05 \\
Follow Me & $50.00 \%$ & 30.66 & 3.31 \\
Indicate Move (Left) & $88.46 \%$ & 24.77 & 3.77 \\
Indicate Move (Right) & $86.36 \%$ & 23.18 & 4.05 \\
Indicate Object (Left) & $57.89 \%$ & 28.21 & 3.74 \\
Indicate Object (Right) & $52.63 \%$ & 35.42 & 3.16 \\
Indicate Stay & $91.30 \%$ & 23.78 & 4.30 \\
Danger & $64.29 \%$ & 32.18 & 3.21 \\
Malfunction & $77.27 \%$ & 29.09 & 3.50 \\
Repeat Previous & $25.00 \%$ & 29.00 & 2.79 \\
\hline Affirmative and Negative perform well, but there are some kinemes, such as \\
Repeat Previous, which are not accurately being identified.
\end{tabular}

Table 5. [Pilot II] Confusion Matrix for Kineme Identification Across All Robots

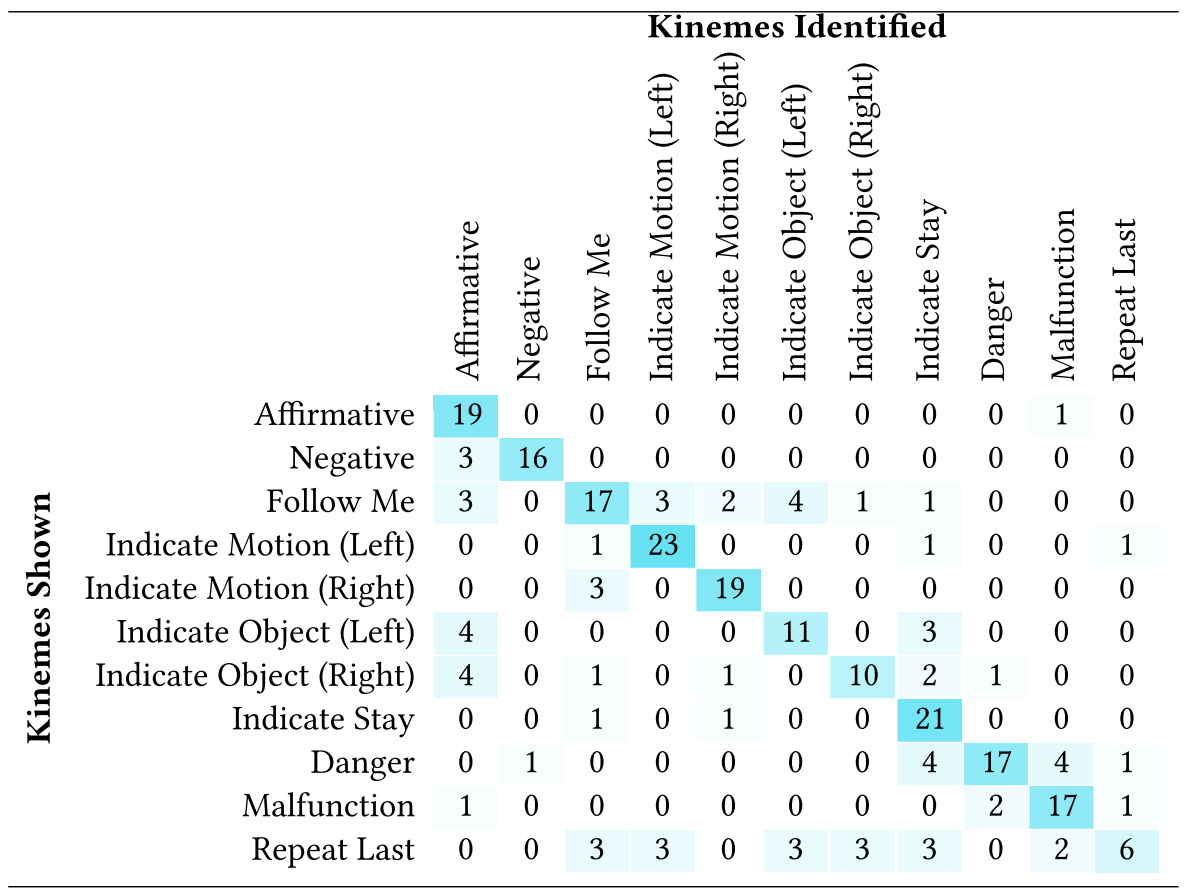

It is apparent that users simply did not remember the Repeat Last kineme, as there is no specific kineme it has been misidentified as.

5.2.3 Participant Opinion. After the completion of their study sessions, participants were given a brief survey measuring their opinions on the kineme systems for each robot. Overall, the Matrice kinemes were rated easiest to use, least confusing, and fastest to use when participants were asked to rank the three systems individually on those metrics. When asked if they felt the kineme systems would be effective at a distance of 10 to 25 feet, all participants responded that the Turtlebot 
kinemes would likely work, $57.1 \%$ responded that the Aqua kinemes would work, and only $28.6 \%$ of participants believed that the Matrice kinemes would work. This belies concern among some participants about the effectiveness of the system at distance. The Aqua kinemes were rated as the slowest and hardest to understand, but only slightly more confusing than Turtlebot2 kinemes. The Turtlebot2 kinemes were considered the most preferable to be used in place of a digital display or speech synthesis system by participants. Participants were moderately sure when they had made mistakes and attributed these mistakes to a combination of confusing kinemes for one another and forgetting the correct response to a kineme. With the exception of the Aqua kinemes, on which participants were unenthusiastic, participants expressed no safety concerns with using any of the systems in an operational environment.

\subsection{Discussion}

The results presented from this pilot study show incremental progress in the successes of individual kinemes and difficulties in the implementation process, particularly for the Aqua kinemes. Although the population was not large enough to test any hypotheses, the results of the full-loop communication testing and user feedback were very useful in directing further development. The poor performance of certain kinemes such as Follow Me led to another development cycle, which solved some of the technical issues hindering recognition. The concerns that participants expressed about the use of kineme systems at distance led to the development of the multi-dimensional study using Amazon MTurk described in Section 3.2, which explores the effectiveness of various communication methods from a number of viewpoints. Last, the success of kinemes as a part of a fullloop interaction system served as further validation of this motion-based communication method, showing that kinemes could work as a part of a system, as well as in isolation.

\section{MULTI-DIMENSIONAL STUDY DESIGN}

With these pilot studies completed, we had evaluated motion-based kineme communication both in simulation and in real-world operation and found evidence that the method would perform acceptably. However, we still had very little information about the capabilities of our kineme communication in comparison to other communication systems on different platforms and from different viewing angles. To explore the relative strengths and weaknesses of these systems for field HRI, we designed a study comparing the four previously described systems (Section 3) on their performance in three different domains and from a variety of viewpoints. Our participantes-recruited using Amazon Mechanical Turk-were trained to use a randomly selected communication system for a randomly selected robot (using a similar education procedure to the EDU2 level from Pilot I and all education from Pilot II), then tested on that system from a randomly selected viewpoint. The conditions of the study are defined by a combination of a randomly selected robot, communication system, and viewpoint, seen in Table 9. Once the participant completed their training, they were asked to complete a brief, unrelated task to distract them before they were tested. For the testing, each participant watched a number of videos of interaction phrases and selected a meaning for the video as well as a confidence rating of that answer while being timed. Each participant was tested on only one condition. This study was conducted in controlled environments, so transferring these precise systems to actual field applications may result in some issues. Nevertheless, the results of this work demonstrate both the strengths and weaknesses of a motion-based communication system and can be used as a basis for further investigation in true field environments, in which studies of this type would be prohibitively expensive and time consuming. This section further describes the details of the study design and administration. This study was submitted for determination to the University of Minnesota's Institutional Review Board and determined to be Not Human Research, making it exempt from full review and continuing review. 


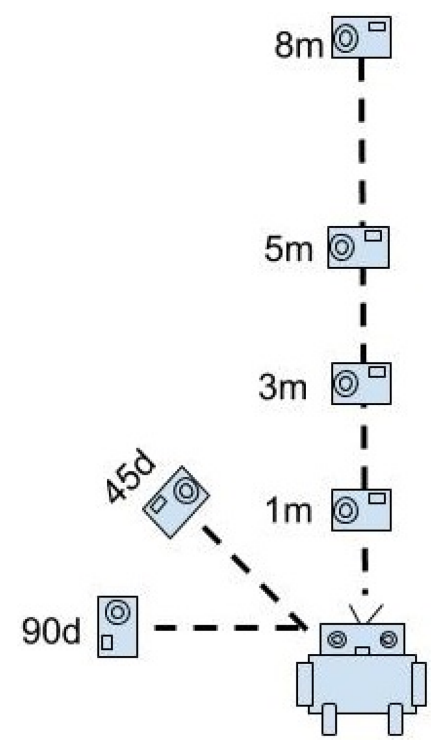

Fig. 8. [MTurk Study] A diagram of the viewpoints captured for the multi-dimensional study.

\subsection{Research Questions}

Due to the large number of experimental conditions in this study, rather than a set of hypotheses, we present the following research questions to guide our exploration:

RQ1: How will the robot/domain affect the performance of each communication system?

RQ2: How will changing viewpoints affect the performance of each communication system?

$R Q 3:$ How will the content being expressed affect the performance of each communication system?

\subsection{Video Data Collection and Viewpoints}

To prepare videos for our survey, each communication system was recorded from a variety of viewpoints. These viewpoints were $3 \mathrm{~m}, 8 \mathrm{~m}, 3 \mathrm{~m}$ at an angle of 45 degrees, and $3 \mathrm{~m}$ at an angle of 90 degrees, diagramed in Figure 8. In addition, an ideal viewpoint for each communication system was selected to be used for the training. This viewpoint, referred to as the EDU viewpoint or education viewpoint, was also a possible viewpoint condition for testing. The EDU viewpoint was selected by choosing the closest head-on distance at which all phrases are entirely visible. For this reason, it was defined as a distance of $1 \mathrm{~m}$ for every system except the Turtlebot and Aqua kineme systems, where a 5-m distance was used. This is because although every other system can be seen in its entirety at a 1-m distance, some Aqua and Turtlebot kinemes require more distance from the camera to be viewed fully. Not every viewpoint was tested for every system because some results are obvious. For instance, the LCD screen cannot be seen from a 90-degree angle, so accuracy should be close to 0 . This self occlusion effect is also present at a 45 -degree angle but is not as pronounced.

For this reason, we elected to test that viewpoint. Similarly, the LED system experiences some self-occlusion at 90 degrees, which make recognition at that viewpoint challenging, but not so challenging as to be entirely worthless for testing. The viewpoint and robot/domain combinations selected for testing can be seen in Figure 9. To capture videos of the systems at every viewpoint, several recording sessions were completed, using two GoPro HERO5 Black cameras, with a 


\begin{tabular}{|c|c|c|c|c|c|c|c|}
\hline & System & EDU & $3 m$ & $8 \mathrm{~m}$ & $45^{\circ}$ & $90^{\circ}$ & Total \\
\hline \multirow{4}{*}{ 岁 } & AUDIO & 8 & 10 & 10 & 0 & 0 & 28 \\
\hline & KINEME & 7 & 8 & 7 & 7 & 7 & 36 \\
\hline & LCD & 7 & 8 & 0 & 6 & 0 & 21 \\
\hline & LED & 7 & 8 & 7 & 7 & 7 & 36 \\
\hline \multirow{4}{*}{ 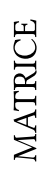 } & AUDIO & 8 & 6 & 9 & 0 & 0 & 23 \\
\hline & KINEME & 7 & 7 & 8 & 7 & 7 & 36 \\
\hline & LCD & 7 & 7 & 0 & 7 & 0 & 21 \\
\hline & LED & 8 & 10 & 7 & 7 & 9 & 41 \\
\hline \multirow{5}{*}{$\begin{array}{l}\stackrel{N}{0} \\
\stackrel{0}{\oplus}\end{array}$} & AUDIO & 8 & 7 & 7 & 0 & 0 & 22 \\
\hline & KINEME & 7 & 7 & 7 & 7 & 8 & 36 \\
\hline & LCD & 7 & 7 & 0 & 7 & 0 & 21 \\
\hline & LED & 7 & 7 & 7 & 8 & 7 & 36 \\
\hline & Total & 88 & 92 & 69 & 63 & 45 & 357 \\
\hline
\end{tabular}

Fig. 9. [MTurk Study] Number of study participants per condition (red intentionally not tested).

1,080-p resolution in the linear aspect ratio (which largely eliminates fish-eye effects caused by the GoPro's wide angle lens) at 24 frames per second. Whenever possible, two views were recorded simultaneously.

Due to scheduling constraints, the Aqua Audio system had to be recorded in a different pool than the rest of the Aqua systems. Therefore, visual clips from the original pool were layered over the audio from the other pool to maintain a visual similarity. Due to this, the speaker is not visible in the Aqua Audio clips. Additionally, the Matrice 100 was placed stationary on a table for the recording of its videos, to allow stable video to be captured of the gimbal, the only part of the drone employed for kinematic communication in this work. This was done, following similar practices in previous work $[3,59]$, to allow the motion of the camera gimbal to be isolated and tested on its own, without the additional motion of the drone in flight. Although there are clearly some confounding factors that would reduce usability of the tested communication systems in comparison between flying and non-flying operation, we believe that the majority of the results will hold up to further inspection.

\subsection{Participant Recruitment and Management}

Participants, recruited using Amazon Mechanical Turk, were required to be in the United States and to have completed more than 5,000 Human Intelligence Tasks with an approval rating of 97\% or greater. Additionally, participants were marked with a qualification after completing the survey, which prevented multiple survey submissions from the same person. Mechanical Turk was used to gather a more diverse population in terms of age and geographical location within the United States, diverging from the college student populations used for Pilots I and II. The $\mathrm{Hu}-$ man Intelligence Tasks posted on Mechanical Turk paid participants $\$ 1.50$ and was estimated to take an average of 12 to 15 minutes, meaning that users would be paid approximately minimum wage. Submissions were typically approved within 24 to 48 hours of completion and would have been auto-approved if left untouched for 7 days. To prevent time and data loss due to any issues, participants were recruited in 16 batches of varying sizes, the majority being of 25 participants. Although conditions were randomly assigned, groups were balanced to have approximately seven participants testing each robot/system/viewpoint combination. The only criteria for accepting 


\section{Participant Gender}

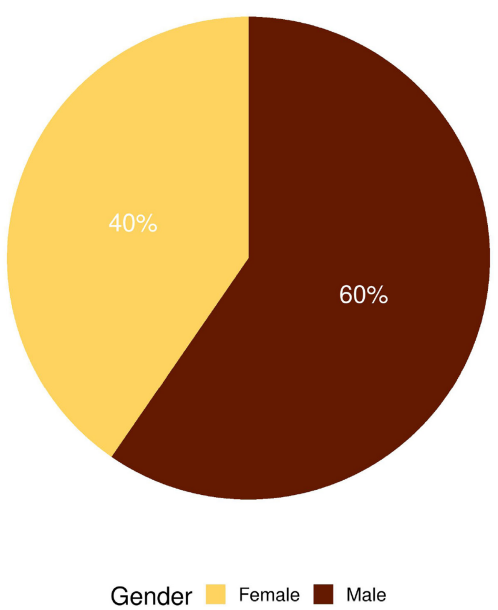

(a) Participant Gender

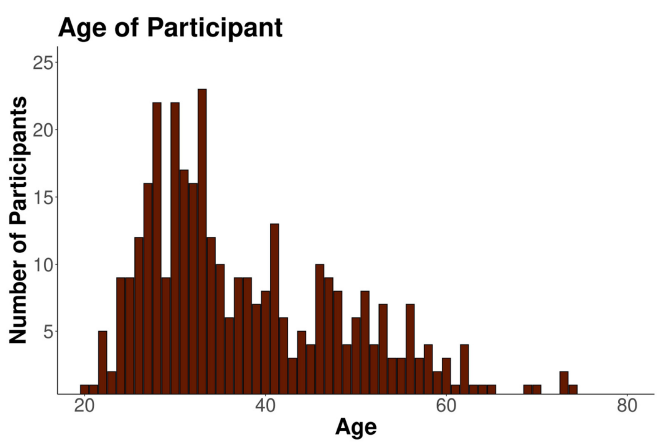

(c) Participant Age

\section{Participant Race}

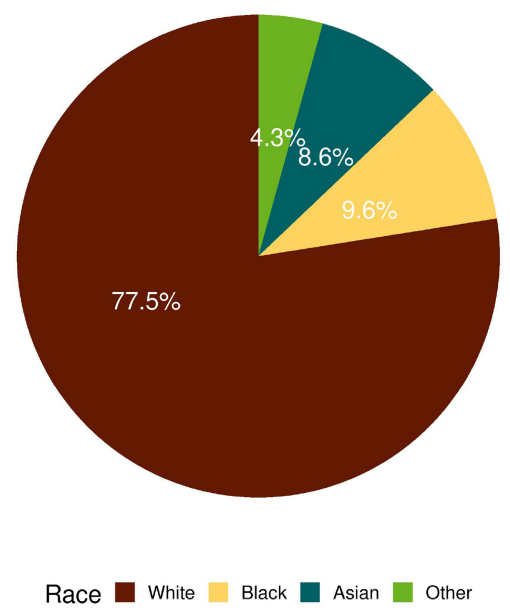

(b) Participant Race

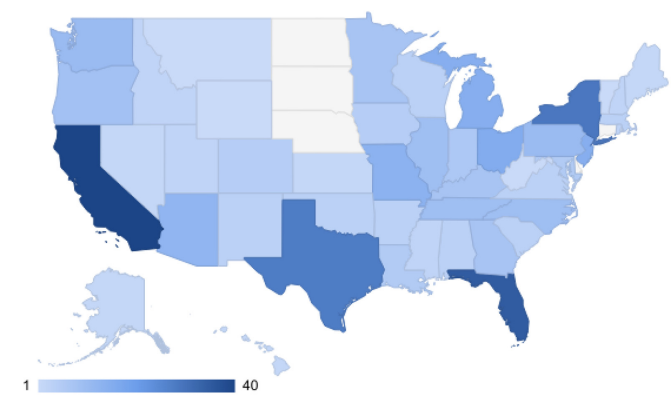

(d) Participant State of Residence

Fig. 10. [MTurk Study] Demographic results for the population of the MTurk study, showing a relatively well balanced sample of people across the United States, mostly younger than 40 years, mostly white, and slightly more men than women.

Mechanical Turk work and paying the worker was that users spend an amount of time on the educational page equal to at least $25 \%$ of the duration of the education video. Users who spent less than a quarter of the video's duration before continuing were considered to have made a bad-faith effort and were rejected. They were, however, paid $\$ 0.75$ (half-pay) for their time. The line for inclusion in the dataset was set higher: only users who spent at least $75 \%$ of the video duration on the page were included in the analysis.

\subsection{Population Statistics}

The population $(\mathrm{N}=357)$ for this study was relatively diverse. We surveyed 386 participants, 29 of whom were excluded from all analyses due to inadequate education video watch times. Some information about the population including age, race, gender, and state of residence can be found in Figure 10 . The 357 included participants were $58 \%$ male and $42 \%$ female and had largely completed 
a 4-year bachelor's degree as their highest level of education. Although $44.9 \%$ had their bachelor's degrees, $11.8 \%$ had some higher degree (masters, Ph.D., MD), and $42.5 \%$ had a high school diploma, an associate's degree, or some college experience. Less than $1 \%$ had no high school diploma. Most (91.1\%) were employed, with $12.8 \%$ self-employed within that figure. Participants came from all over the United States, hailing from 45 of the 50 states, with the top three being California (11.3\%), Florida (9.0\%), and New York (7.3\%). Last, participants covered a wide range of ages. The mean age was 37 years, with a standard deviation of 10.9 years, a minimum recorded age of 20 , and a maximum recorded age of 74 . It is clear from these demographics that our population is diverse in age and location in the United States.

\subsection{Education and Distraction Procedure}

Once participants entered the survey and passed a bot check, they were randomly assigned a condition and directed to an education procedure. The education procedure for each condition consisted of a video composed of the 14 interaction phrases in a set order from the EDU (ideal) viewpoint of the robot, using the system of the participant's condition. Participants were asked to watch the entire video without skipping around and warned that their payment would depend on watching the entire video, although they were permitted to leave the page at any time. As mentioned previously, only participants who spent at least $75 \%$ of the duration of their education video on the page were included in the analysis. Following the education procedure, participants were asked to solve five ninth-grade-level mathematics questions as a distraction procedure. Distraction procedures are a common method in psychology research used to induce forgetfulness in subjects. Some examples can be found in the 1950's memory research of Brown [13] and Peterson and Peterson [46], or other more recent work on working memory by Waris et al. [60]. In our work, a distraction procedure is used to separate the training and testing phases of the study so that participants are less likely to be able to hold the entirety of the training they just completed in their short-term memory. The training in this study was similar to the EDU2 level of education used in Pilot I (see Section 4.1.3), intended to replicate a full but unpracticed knowledge of the system, but not expert-level training. This level of training was intentional, as experts would likely have achieved similar accuracy and speed of system use regardless of system or viewpoint. Intentionally limiting participants to a more casual knowledge of the system they were about to test was intended to aide in revealing the comparative strengths and weaknesses of the communication system studied.

\subsection{Testing and Evaluation}

Once participants had completed their education and distraction procedures, the testing portion of the survey began. Each participant was shown videos of all 14 interaction phrases in a random order, using their robot and system combination from the viewpoint indicated in their condition. Some received the EDU viewpoint as their testing viewpoint, so they had the same viewpoint for education and testing. For each video, the participant was shown a video hosted on YouTube and asked to select its meaning from a drop-down menu. The time from when the participant entered the webpage to when they left it was recorded. If a meaning was selected, they were then asked what their confidence in their choice was, on an ordinal scale from 1 to 10 (10 being the most confident). This scale was increased from a range from 0 to 5 to a range from 0 to 10 for this study in an attempt to capture more nuance in the results of the confidence question. Participants were also given the option to select "Unable to select meaning." If they selected this option, the confidence question was not presented, and they were instead asked what made them unable to make a selection: forgetting the meaning, being unable to see the interaction phrase, the survey not displaying the video, or some other issue. Once participants had completed each video, they 
Table 6. [MTurk Study] Confusion Matrices for Every Communication System

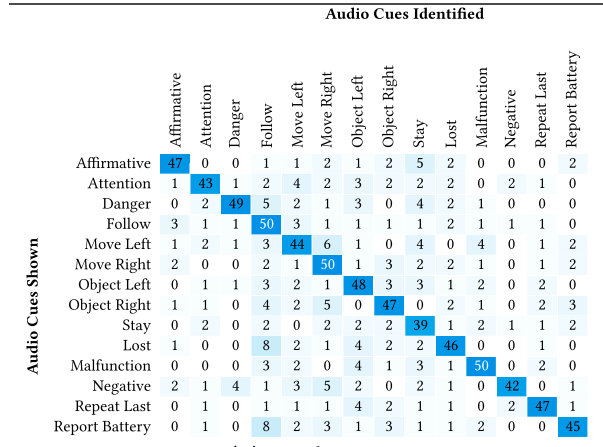

(a) Audio system

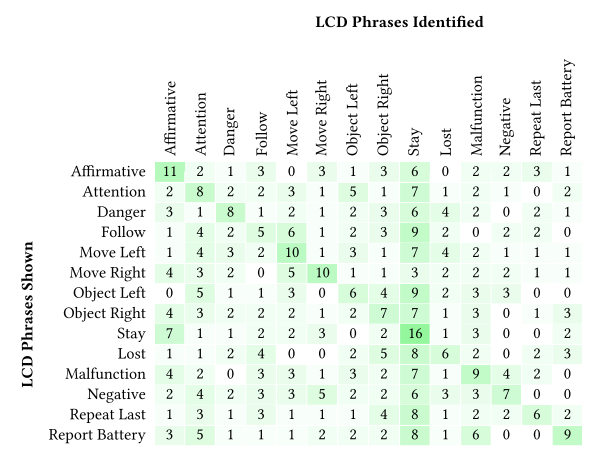

(c) LCD system

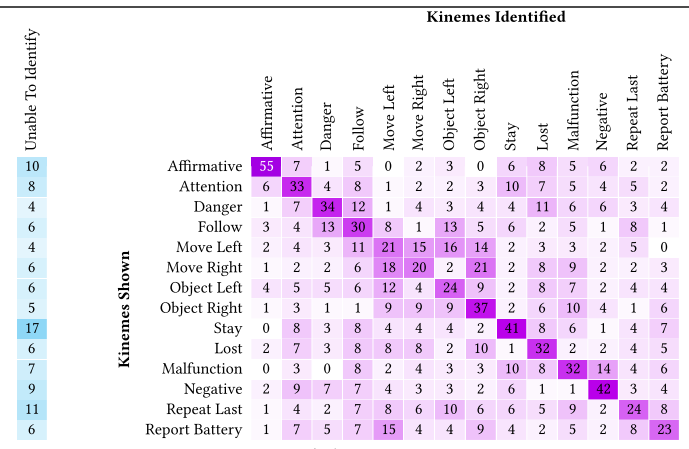

(b) Kineme system

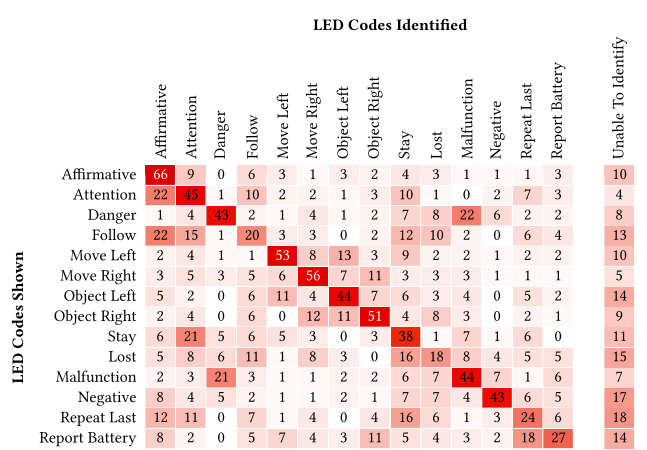

(d) LED system

The audio system shows low confusion, with no specific misidentification pattern. The kineme system shows moderate confusion, with a pattern of confusion around directional kinemes. The LCD system has a broad, scattered confusion pattern, with high rates of users marking their answer as "Unable to Identify." The LED system has moderate confusion, with the misidentification happening around directional codes and codes that share colors.

were given a brief survey on their opinions of the communication method they had just tested, then given a completion code to submit on Mechanical Turk for payment.

\section{RESULTS}

\subsection{Comparison Metrics}

The four metrics used to compare the effectiveness of the communication systems are accuracy, operational accuracy, confidence, and time to answer. Accuracy is the correctness of a participant's selected answer, measured absolutely with no partially correct answers. Operational accuracy is the same metric but only considers answers rated a 5 or higher in confidence, to simulate the answers that a user would be likely to act upon. Confidence and time to answer are simply the values recorded from the confidence question and the time participants took to select a meaning for a video. However, the time to answer data was processed to remove outliers by discarding values greater than 150 seconds. This was set as the cutoff because for all interaction phrases, 95\% of responses had a time to answer lower than 150 seconds (mean 95th percentile was 76.78 seconds). Durations of these outlier answers greatly exceeded 150 seconds (e.g., 500 seconds or greater), which suggests that the webpage was left open while the participant did something else. 


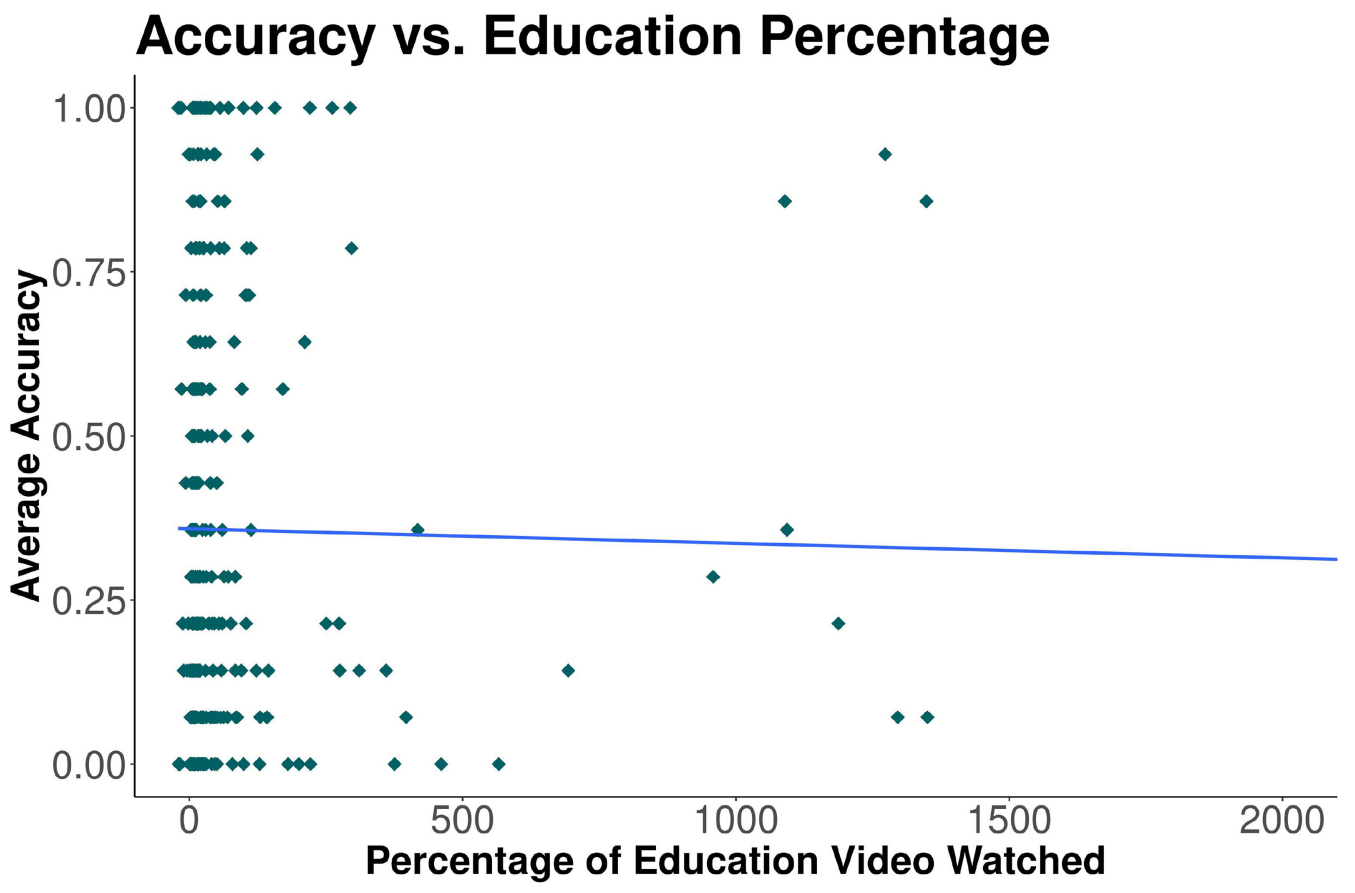

Fig. 11. [MTurk Study] Internal validity results comparing average accuracy with the percentage difference between the education video's duration and the time spent watching it. Each point represents a participant's accuracy vs. the percentage of the education video they watched. If a participant spent a longer time on the page than the duration of the video, their percentage of the video watched will be greater than $100 \%$. The trend line shows no significant effect.

\subsection{Internal Validity}

Several statistical tests were run to check for systematic bias in the study, at a confidence level of $\alpha=0.01$, which is used for all tests in this analysis. A ranked correlation test using Spearman's $\rho$ found no significant relationship between the percentage of education video that a participant watched and their average accuracy in testing $(p=0.184, \rho=0.0704)$, as shown in Figure 11. An ANOVA test similarly found no significant relationship between the level of formal education completed and average accuracy $(F(1,356)=0.359, p=0.167)$. We also found no relationship between average accuracy and the batch within which a participant was recruited $(F(1,356)=$ $0.181, p=0.241)$. Finally, no statistically significant difference in average accuracy was found between male and female participants when using a $t$-test $(p=0.725, d=0.0374)$. No significant threats to internal validity were detected.

However, accuracy was significantly lower for all systems than expected. For instance, accuracy for the audio device for all robots at the EDU viewpoint was 64\%, and accuracy was no higher than $72 \%$ for any individual robot audio instance despite the fact that the audio system simply plays English phrases over a speaker. Additionally, an earlier study of kinemes for the same three robots used in this study achieved greater than $60 \%$ accuracy for each kineme system. The method of testing in this study is lower fidelity, with videos on a screen serving as a worse testing media than physical observation of the robot. This is an unfortunate effect of the logistical considerations prompted by the size of this study (some 350 participants, three different independent variables, etc.). Although the exact values for accuracy may be somewhat lower than expected, we believe 
Table 7. [MTurk Study] Systems Compared by Robot (Best Values Are Bolded)

\begin{tabular}{|c|c|c|c|c|c|}
\hline & Robot & Acc. & Op_Acc. & Conf. & $\overline{\text { Time (s) }}$ \\
\hline \multirow{3}{*}{$\stackrel{\circ}{\stackrel{0}{\beta}}$} & Aqua & $19.6 \%$ & $15.6 \%$ & 4.4 & 20.7 \\
\hline & Matrice & $81.1 \%$ & $78.9 \%$ & 8.9 & 16.9 \\
\hline & Turtlebot & $85.1 \%$ & $84.7 \%$ & 9.2 & 18.3 \\
\hline \multirow{3}{*}{$\underset{⿱ ㇒}{\mid}$} & Aqua & $25.6 \%$ & $19.6 \%$ & 4.5 & 35.0 \\
\hline & Matrice & $34.3 \%$ & $27.0 \%$ & 4.8 & 32.1 \\
\hline & Turtlebot & $24.8 \%$ & $20.0 \%$ & 4.7 & 36.7 \\
\hline \multirow{3}{*}{ తి } & Aqua & $27.9 \%$ & $26.5 \%$ & 4.9 & 24.5 \\
\hline & Matrice & $7.8 \%$ & $2.0 \%$ & 2.1 & 17.9 \\
\hline & Turtlebot & $2.4 \%$ & $2.0 \%$ & 1.9 & 16.8 \\
\hline \multirow{3}{*}{ 곡 } & Aqua & $33.7 \%$ & $25.4 \%$ & 4.7 & 23.7 \\
\hline & Matrice & $35.9 \%$ & $28.0 \%$ & 4.9 & 24.6 \\
\hline & Turtlebot & $35.3 \%$ & $28.4 \%$ & 5.0 & 22.3 \\
\hline
\end{tabular}

Accuracy rates for the Audio and LCD systems are very robot dependent (likely due to the differences between underwater and in-lab conditions). The LED and Kineme systems achieve similar accuracy in each scenario.

that the general trend of results should be correct, and statistically large effects should persist in in-person testing.

\subsection{Robot Comparisons}

Our first point of comparison is the robot assigned to the participant's condition, which relates to RQ1. We find through ANOVA tests that robot selection causes a small but statistically significant effect on accuracy $(F(1,356)=0.154, p=0.00962)$ and operational accuracy $(F(1,356)=0.158$, $p=0.00675$ ) when considering all systems together (Table 7). Pairwise Games-Howell tests find that the effect on accuracy is between the Aqua platform and the other two platforms, with no significant effect between the Turtlebot and Matrice, for average accuracy and operational accuracy both. This confirms our expectations that underwater communication is challenging, at least in our test environments. Further, this effect appears to be mostly from the audio system. When considering only the audio system, large significant effects are found from robot selection in accu$\operatorname{racy}(F(1,72)=1.37, p<0.001)$, confidence $(F(1,72)=1.11, p<0.001)$, and operational accuracy $(F(1,72)=1.31, p<0.001)$ using ranked ANOVA tests. This result can be explained by the simple fact that sound is much clearer in the air than underwater. Although sound waves propagate far underwater, they are often garbled and difficult to hear, along with being distorted by background sounds. This effect is not seen for the other systems, as they rely on the visibility of the communication vector, which is approximately the same in the air and the pool water used for this study. Even though the effect is not significant, Aqua LCD also has greater average accuracy than the same system on other robots. This is likely due to the nature of the lighting in the pool environment, which makes the LCD easier to read (at short distances) than in air.

\subsection{Viewpoint Comparisons}

To explore RQ2, we consider the relationships that viewpoint has on our measured variables for each system. Statistically significant relationships are found via ANOVA testing between changing 
Table 8. [MTurk Study] Systems by Viewpoint

\begin{tabular}{|c|c|c|c|c|c|}
\hline & Viewpoint & Acc. & Op_Acc. & Conf. & Time (s) \\
\hline \multirow{3}{*}{ 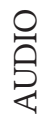 } & EDU & $79.8 \%$ & $78.9 \%$ & 8.4 & 19.1 \\
\hline & $3 \mathrm{~m}$ & $59.0 \%$ & $55.9 \%$ & 7.3 & 17.6 \\
\hline & $8 \mathrm{~m}$ & $51.9 \%$ & $48.6 \%$ & 6.2 & 19.5 \\
\hline \multirow{5}{*}{ 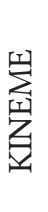 } & EDU & $35.7 \%$ & $28.2 \%$ & 5.1 & 36.7 \\
\hline & $3 \mathrm{~m}$ & $32.1 \%$ & $27.6 \%$ & 5.5 & 35.5 \\
\hline & $8 \mathrm{~m}$ & $32.5 \%$ & $24.0 \%$ & 4.2 & 35.9 \\
\hline & 45 degrees & $23.1 \%$ & $17.3 \%$ & 4.2 & 31.2 \\
\hline & 90 degrees & $24.7 \%$ & $19.5 \%$ & 4.2 & 33.4 \\
\hline \multirow{3}{*}{ తి } & EDU & $32.3 \%$ & $27.9 \%$ & 3.9 & 21.1 \\
\hline & $3 \mathrm{~m}$ & $4.2 \%$ & $1.3 \%$ & 2.1 & 20 \\
\hline & 45 degrees & $3.6 \%$ & $2.5 \%$ & 2.9 & 18 \\
\hline \multirow{5}{*}{ 龱 } & EDU & $61.4 \%$ & $51.9 \%$ & 6.2 & 22 \\
\hline & $3 \mathrm{~m}$ & $46.6 \%$ & $34.6 \%$ & 5.1 & 22.5 \\
\hline & $8 \mathrm{~m}$ & $17.7 \%$ & $11.9 \%$ & 3.9 & 23.3 \\
\hline & 45 degrees & $34.4 \%$ & $26.3 \%$ & 5.6 & 24.9 \\
\hline & 90 degrees & $19.3 \%$ & $13.4 \%$ & 3.6 & 25.2 \\
\hline
\end{tabular}

Kineme accuracy changes the least of all systems in response to change in viewpoint.

Best values are bolded.

Table 9. [MTurk Study] System Performance by Viewpoint and Robot

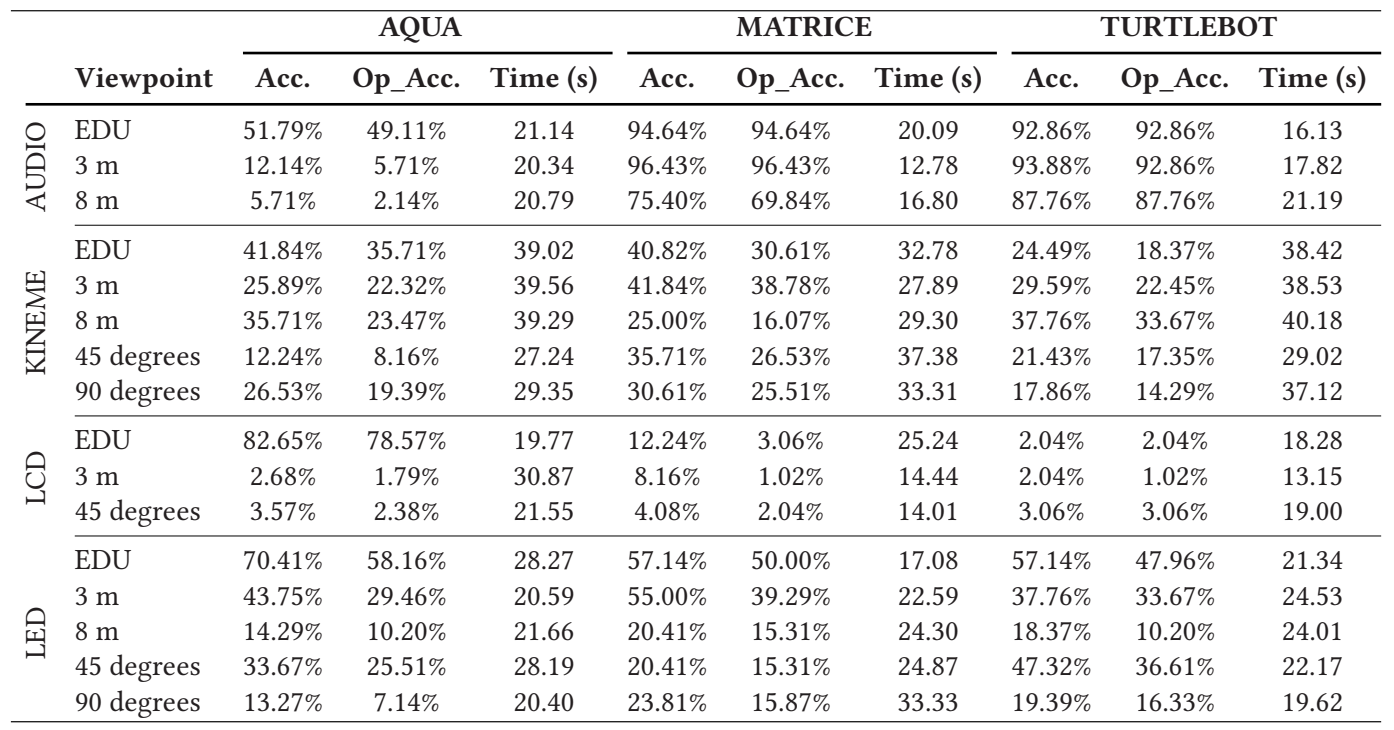

Note some domain-specific effects of viewpoints. Audio accuracy crashes for Aqua but does not change as much for Matrice and Turtlebot. Meanwhile, LCD works exceptionally well for Aqua at the EDU viewpoint because of the light conditions but still falls dramatically at other viewpoints. 
viewpoint and effects on accuracy $(F(1,356)=0.384, p<0.00001)$ and operational accuracy $(F(1,356)=0.380, p<0.00001))$, and confidence $(F(1,356)=0.250, p=0.0000553)$, but not time to answer $(F(1,356)=0.135, p=0.227)$, when considering all the systems together. The effect per system can be observed mostly in the LED and audio systems, which can be seen more clearly in Table 9. This is due to the fact that although the audio and LEDs are easy to recognize at close viewpoints, they become increasingly difficult to recognize at further and more rotated viewpoints. However, the kineme system does not suffer from these effects as severely.

When further inspecting the relationships between different systems and viewpoints, we find that the 8-m, 45-degree, or 90-degree viewpoints are most frequently involved in relationships with significant differences in accuracy or operational accuracy between other viewpoints in the same system. Using unranked pairwise Games-Howell testing [25] to examine the specific relationships between the accuracy and operational accuracy of communication systems at different viewpoints, we find statistically significant effects on both accuracy and operational accuracy for our baseline systems. For the audio system, the EDU and 8-m viewpoints have significant differences in both accuracy ( $p=0.0248, f=-0.773)$ and operational accuracy ( $p=0.0189, f=-0.804)$. In the case of the LCD system, we find significant reductions in accuracy between the EDU viewpoint and the 3-m viewpoint $(p=0.011, f=-1.03$ ) as well as between the EDU viewpoint and 45-degree viewpoint $(p=0.009, f=-1.02)$. We find similar reductions in accuracy for the LED system for 5 of the 10 pairwise viewpoint comparisons, particularly when comparing the EDU viewpoint with $8 \mathrm{~m}(p=0.001, f=-2.106), 45$ degrees $(p=0.008, f=-1.095)$, and 90 degrees $(p=0.001$, $f=-1.967)$.

However, when we perform the same testing on kineme accuracy, we find no statistically significant differences in accuracy or operational accuracy between any viewpoints. Even the most challenging viewpoints compared the EDU viewpoint do not reach a level of accuracy difference that can be considered statistically significant: EDU vs. $8 \mathrm{~m}(p=0.00, f=-0.220)$, EDU vs 90 degrees $(p=0.432, f=-0.538)$. Although the overall accuracy of the kineme method is low, as the accuracy is much higher than that of a random guess (7\%), we suggest that this shows that kinemes are more viewpoint invariant than other systems. This effect is not as pronounced when considering the viewpoint performance of each robot's system separately, but it is still present. Every communication system and robot combination experiences a differing amount of effect from viewpoint, but kineme systems remain affected less than other systems in most cases. This data reveals a possible feature of motion-based communication: greater viewpoint invariance than other modalities. Given the limitations of this data, it should not be assumed that any motion-based communication system will experience minimal effects from differing viewpoints, but with further research including more high-fidelity testing, it may be possible to determine the extent to which this viewpoint invariance exists, both for these individual systems and for generic motion communication systems.

\subsection{Content Comparisons}

To explore the effect of message content on communication with our four systems (RQ3), we consider three categories of our interaction phrases. As seen in Table 1, we separate the interaction phrases into three types: Conversational (managing interaction state), Directional (relating to the physical world and things or vectors in it), and Complex (relating to more abstract concepts). Generally, the accuracy, operational accuracy, and time to answer follow the same trends found when looking at the systems overall: the audio system is the best, LCD is the worst, and the kineme and LED systems are nearly tied. An interesting point can be observed in the effect of content on the accuracy of the systems (seen in Table 10). Here we can see that although kinemes and LEDs have similar accuracies for conversational and complex interaction phrases, the accuracy of LEDs is significantly higher for directional phrases. A ranked pairwise Games-Howell [25] test confirms this, 
Table 10. System Accuracy Compared by Phrase Content

\begin{tabular}{|c|c|c|c|c|c|}
\hline & Viewpoint & Acc. & Op_Acc. & Conf. & Time (s) \\
\hline \multirow{3}{*}{ 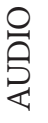 } & Conversational & $61.0 \%$ & $44.0 \%$ & 7.17 & 19 \\
\hline & Directional & $62.5 \%$ & $61.0 \%$ & 7.2 & 19.6 \\
\hline & Complex & $65.8 \%$ & $62.0 \%$ & 5.73 & 17.3 \\
\hline \multirow{3}{*}{ 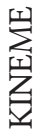 } & Conversational & $36.0 \%$ & $26.0 \%$ & 5.04 & 31.5 \\
\hline & Directional & $26.5 \%$ & $19.9 \%$ & 4.54 & 35.2 \\
\hline & Complex & $28.0 \%$ & $21.5 \%$ & 3.4 & 36.7 \\
\hline \multirow{3}{*}{ తి } & Conversational & $13.0 \%$ & $8.0 \%$ & 2.91 & 19 \\
\hline & Directional & $15.6 \%$ & $10.3 \%$ & 2.97 & 19.7 \\
\hline & Complex & $11.7 \%$ & $11.9 \%$ & 2.21 & 20.5 \\
\hline \multirow{3}{*}{ 至 } & Conversational & $39.0 \%$ & $27.0 \%$ & 4.7 & 23.5 \\
\hline & Directional & $42.8 \%$ & $31.6 \%$ & 5.05 & 23.3 \\
\hline & Complex & $26.9 \%$ & $21.0 \%$ & 3.52 & 24.2 \\
\hline
\end{tabular}

LEDs have high accuracy at communicating directional phrases, whereas kinemes do not have the same benefit (possibly due to participants confusing Left kinemes for Right ones, whether to use their directions or those of the robot).

showing a strongly significant relationship between the two systems and accuracy of directional phrases ( $p=0.001, f=0.0 .514$ ). This suggests that although kinemes are strongly spatial, LEDs were more effective in expressing directional information. The directional LED codes made use of a feature of the LED system that no other interaction phrases made: they implied motion in the direction indicated by flashing the lights in a series, toward the point of interest. One possible reason for the lack of similar performance in the kineme system can be seen in Table 6, where we can see frequent misidentifications within the directional kinemes. The directional kinemes mostly begin with facing the robot in the indicated direction, which is likely leading to misidentifications.

\subsection{Opinion Survey}

In the last portion of the survey, participants were asked to rate the robot and system combination they had been tested on from effective to ineffective on a number of qualities: pleasantness, naturalness, speed, and accuracy of the communication. The Matrice version of kinemes and both of the non-aquatic audio systems are often rated as effective for accuracy, whereas both the Matrice and Turtlebot LCDs are rated as ineffective. The LED systems are rated as effective for speed of communication, whereas the kineme systems typically are rated lower. For naturalness, the kinemes of Aqua and the Matrice are rated as effective along with the audio systems, whereas the LCD systems are often rated as ineffective. The full results can be seen in Table 11.

\subsection{Discussion}

The complex nature of this study makes the results somewhat difficult to understand. In particular, the combinatory nature of the variables studied makes it difficult to draw a single conclusion from the results on them. Generally, we find that robot choice does not make a significant difference in the overall effectiveness of communication systems. The exception is the audio system, which has the greatest domain differences (due to propagation of sound). This suggests that although most of our communication systems could be applied with similar effectiveness in most domains, the amount of distortion of their media may cause issues. In this case, the distortion of media is 
Table 11. [MTurk Study] User Opinions of the Robot and System Combination They Tested

\begin{tabular}{|c|c|c|c|c|c|c|c|c|c|c|c|c|c|}
\hline & & \multicolumn{3}{|c|}{ Accuracy } & \multicolumn{3}{|c|}{ Speed } & \multicolumn{3}{|c|}{ Naturalness } & \multicolumn{3}{|c|}{ Pleasantness } \\
\hline & & Effective & Neutral & Ineffective & Effective & Neutral & Ineffective & Effective & Neutral & Ineffective & Effective & Neutral & Ineffective \\
\hline \multirow{4}{*}{$\begin{array}{l}\overleftrightarrow{S} \\
\text { Q્વ }\end{array}$} & Audio & $25.0 \%$ & $21.4 \%$ & $53.6 \%$ & $44.4 \%$ & $33.3 \%$ & $22.2 \%$ & $28.6 \%$ & $25.0 \%$ & $46.4 \%$ & $25.0 \%$ & $42.9 \%$ & $32.1 \%$ \\
\hline & Kineme & $27.8 \%$ & $36.1 \%$ & $36.1 \%$ & $25.0 \%$ & $38.9 \%$ & $36.1 \%$ & $22.2 \%$ & $33.3 \%$ & $44.4 \%$ & $16.7 \%$ & $19.4 \%$ & $63.9 \%$ \\
\hline & LCD & $42.9 \%$ & $23.8 \%$ & $33.3 \%$ & $28.6 \%$ & $33.3 \%$ & $38.1 \%$ & $14.3 \%$ & $42.9 \%$ & $42.9 \%$ & $14.3 \%$ & $52.4 \%$ & $33.3 \%$ \\
\hline & LED & $30.6 \%$ & $36.1 \%$ & $33.3 \%$ & $52.8 \%$ & $33.3 \%$ & $13.9 \%$ & $16.7 \%$ & $36.1 \%$ & $47.2 \%$ & $27.8 \%$ & $38.9 \%$ & $33.3 \%$ \\
\hline \multirow{4}{*}{ 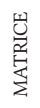 } & Audio & $82.6 \%$ & $13.0 \%$ & $4.3 \%$ & $69.6 \%$ & $21.7 \%$ & $8.7 \%$ & $43.5 \%$ & $52.2 \%$ & $4.3 \%$ & $65.2 \%$ & $4.3 \%$ & $30.4 \%$ \\
\hline & Kineme & $61.1 \%$ & $27.8 \%$ & $11.1 \%$ & $38.9 \%$ & $33.3 \%$ & $27.8 \%$ & $41.7 \%$ & $27.8 \%$ & $30.6 \%$ & $63.9 \%$ & $11.1 \%$ & $25.0 \%$ \\
\hline & LCD & $4.8 \%$ & $23.8 \%$ & $71.4 \%$ & $14.3 \%$ & $28.6 \%$ & $\mathbf{5 7 . 1 \%}$ & $0.0 \%$ & $47.6 \%$ & $52.4 \%$ & $0.0 \%$ & $61.9 \%$ & $38.1 \%$ \\
\hline & LED & $43.9 \%$ & $31.7 \%$ & $24.4 \%$ & $56.1 \%$ & $29.3 \%$ & $14.6 \%$ & $24.4 \%$ & $41.5 \%$ & $34.1 \%$ & $29.3 \%$ & $29.3 \%$ & $41.5 \%$ \\
\hline \multirow{4}{*}{ 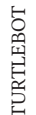 } & Audio & $90.9 \%$ & $9.1 \%$ & $0.0 \%$ & $86.4 \%$ & $13.6 \%$ & $0.0 \%$ & $52.4 \%$ & $42.9 \%$ & $4.8 \%$ & $59.1 \%$ & $9.1 \%$ & $31.8 \%$ \\
\hline & Kineme & $36.1 \%$ & $33.3 \%$ & $30.6 \%$ & $36.1 \%$ & $30.6 \%$ & $33.3 \%$ & $16.7 \%$ & $36.1 \%$ & $47.2 \%$ & $19.4 \%$ & $36.1 \%$ & $44.4 \%$ \\
\hline & LCD & $14.3 \%$ & $23.8 \%$ & $61.9 \%$ & $9.5 \%$ & $28.6 \%$ & $61.9 \%$ & $14.3 \%$ & $23.8 \%$ & $61.9 \%$ & $19.0 \%$ & $57.1 \%$ & $23.8 \%$ \\
\hline & LED & $44.4 \%$ & $19.4 \%$ & $36.1 \%$ & $52.8 \%$ & $13.9 \%$ & $33.3 \%$ & $13.9 \%$ & $47.2 \%$ & $38.9 \%$ & $25.0 \%$ & $33.3 \%$ & $41.7 \%$ \\
\hline
\end{tabular}

The system with the highest percentage selection of effective and ineffective for each robot is shown in bold. For the Aqua robot, for instance, LCD is most often selected as effective for accuracy and audio most often selected as ineffective.

the garbling of sound through water, but in another use case, it could be the distortion of visual information.

We also find that, generally, viewpoints affect communication success as expected: the further away and more rotated from the "standard" view, the more systems fail. The exception here is kinemes that appear somewhat invariant to viewpoint changes in terms of their accuracy. This result echoes our previous findings in Pilot I, where $66.7 \%$ of users reported that they would prefer the kineme system to an LED or LCD system at a significant distance. Expanded interaction distance and angles is potentially a significant benefit of kinemes, but they apply only insofar as the interactant can still see the robot. In murky water conditions, for instance, this advantage disappears. The LCD method does not see significant viewpoint differences overall, mostly because it fails equally badly at any viewpoint that is not EDU. This suggests that the selection of communication system is innately tied to interactant range and orientation. Although a robot can, and sometimes should, alter its position to enter a more ideal interaction range, it sometimes cannot. In that case, an appropriate system should be selected to avoid low-accuracy communication.

Last, we conducted some analysis on interaction phrase content's effect on system performance, but our results are somewhat inconclusive. An important result from this analysis was the finding that kinemes and LEDs do not have significantly different accuracy, operational accuracy, or confidence for non-directional interaction phrases (Affirmative, Danger, etc.). This implies that the two could be used somewhat interchangeably as long as the content is not directional, in which case LEDs should be used. It also implies that the directional accuracy of kinemes could be improved. Since kinemes exist in 3D space, they should be at least as accurate as LEDs for directional information.

\section{RECOMMENDATIONS AND FUTURE WORK}

\subsection{Recommendations}

Based on the results of our research in this work, we offer a few recommendations in this section, both for researchers interested in replicating or extending these results, and for developers interested in using human-robot communication methods in the field.

8.1.1 Interaction Phrase Selection. To select a library of interaction phrases, we recommend that researchers begin by completing interviews with users of the robot they are developing for, along with application experts. Stemming from these interviews, simple mock interactions can be created in which the environment and task are defined based on interview data. By asking domain 
experts and robot users to complete these tasks with a partner, the exact information for which communication is required can be elicited, providing the researcher with a set of concepts from which to build their interaction phrase library.

8.1.2 Application of Communication Systems by Domain. Based on our results in controlled environments, we suggest that audio (at least in the form of spoken language) not be used for underwater communication, as speech cannot be effectively recognized underwater. We further suggest that LCDs be avoided for environments with a high probability of direct sunlight, and that displays selected for outdoor use be high contrast, to be more easily read.

8.1.3 Kinemes and Challenging Viewpoints. Our results in Section 7.4 suggest that kinemes may have some level of performance invariance to viewpoint changes. Due to this, we recommend the use of kinemes over most other systems when communicating at challenging orientations and distances. Although some other communication methodologies (i.e., LEDs) can be modified to make them more viewpoint invariant, by reducing dead angles and increasing output stimuli, some methodologies (i.e., digital displays) cannot be modified sufficiently to overcome their shortcomings at long distances or deep viewing angles. Therefore, we recommend attempting to design communication systems in such a way that they are visible around the entire robot, and using motion when such modifications are not possible (or to supplement these modifications).

8.1.4 Kinemes and LED Codes and Directionality. Based on our results in Section 7.5, we suggest that LED codes be used to communicate directional concepts. Our results do not indicate that motion cannot communicate directional concepts, so the use of kinemes is also an option. However, LED codes may be the more prudent choice, although improvement of kineme systems may change this result in future. Additionally, different motion-based systems may yield different results. For instance, a gaze cue based motion system in Hart et al. [29] outperformed their baseline LED system in communicating directionality. Clearly, further investigation is needed, but our recommendation would be to use LED codes in general and explore other ways to use motion for communication direction information.

\subsection{Future Work}

Our work on RCVM has already yielded successful communication systems for three robots and a variety of theoretical implications. Of course, expanding RCVM implementations across other robots is an exciting area for future work, as the development of implementations for more robots will continue to expand our understanding of how these languages can be designed. We are currently developing an implementation for the new LoCO AUV [22]. Additionally, expanding the final study presented in this work to an in-person design would be useful to validate the degree to which this work's results will hold for in-person interaction. However, our research on the three robot implementations discussed in this work has also revealed a wide variety of related problems ripe for future exploration. In this section, we will briefly describe two practical future areas of focus (expansion and refinement of kineme language) and three more theoretical issues (the study of kineme learning, adaptation of kinemes to interactant viewpoint, and autonomous communication vector selection).

8.2.1 Expansion of Kineme Language. As mentioned previously, the current RCVM protocol includes 14 kinemes. Some of these kinemes can be used to communicate a range of meanings. For instance, the Directional kineme can communicate a directional focus in any direction the robot is capable of moving. There is, however, a vast configuration space of possible motions of each robot that can be mapped to meanings, a space that is mostly unused. In the future, we plan to 
increase the size of the RCVM protocol, focusing on working closely with actual interactants in field robotics situations to determine what interactions are missing from RCVM as it stands.

8.2.2 Prefix-Free Kinemes. As we consider increasing the number of possible communicated phrases, we must consider the fact that every new kineme has a chance to be confused with another. We saw this in our tests and particularly discovered that kinemes containing another kineme (or something similar) as part of their movement are easily confused. To avoid further kineme confusions as we expand the protocol, we intend to investigate redesigning some kinemes to create a prefix-free protocol where every individual kineme begins differently, so as to improve first-look recognition. Although efforts were made to reduce kineme prefix overlap in the version of kinemes for this work, a truly prefix-free language has not yet been developed for kinemes.

8.2.3 Learning of Kinemes. Pilot I briefly explored the topic of how easy kinemes were to learn and found that increasing education time had a much greater effect on the accuracy and speed of the kineme system than with the comparison LED system. However, this was a very limited educational difference between participants and the overall sample size was quite small. In future work, it would be beneficial to complete longitudinal studies of learning for kinemes to investigate how the skill of recognizing kinemes can be best developed over time.

8.2.4 Adaptation of Kinemes to Viewpoint. Just as the audio communication system could be adapted to greater interaction distances by increasing the output volume of the speaker, it is likely that kineme recognition accuracy could be increased. This could involve increasing the distance covered in a kineme, or simply increasing the intensity of accelerations or "animation" of the gesture. Although this approach (changing output stimuli in response to interaction distance) has been proposed, autonomous systems for doing this with full-body robot gestures are not widespread, and further investigation would be worthwhile.

8.2.5 Communication Vector Selection and Combination. The primary research thread discovered in the work presented in this article is the selection of communication vectors for different tasks. Although our results do show RCVM as a viable communication vector alongside our comparison systems, it is not singularly superior to any other communication system. Due to this, it is desirable for a robot to be able to select what method to use to communicate a concept to an interactant, based on interaction context, environment qualities, communication content, and user preference/capabilities. Additionally, exploring dynamic combinations of multiple communication modalities as a part of this autonomous vector selection process is extremely interesting. The combination of multiple methodologies may help overcome weakness of any individual method but must be balanced to avoid overwhelming the user. Depending on the information being transmitted, the relative position of the interactant, the quality of the environment, and user preference, any number of single or combined communication methodologies might be indicated. In the future, we hope to develop methods for autonomously selecting and combining communication vectors in the field and assess those selections for effectiveness.

\section{CONCLUSION}

RCVM is a protocol, implemented and tested on three different robots, for robot-to-human communication in the field. We developed RCVM to facilitate better underwater field HRI but have since become convinced that RCVM, and in general motion-based communication, has an important role to play in expanding the communication abilities of other field robots. Through our development process, our two small pilot studies, and our multi-dimensional study on Amazon Mechanical Turk, we have found both strengths and weaknesses of the system for which we have also explored solutions. 
Our first pilot established RCVM as a viable communication method that was more easily learned with minimal training than our comparison system, but it left questions about how well it would perform in the real world, given that it was tested only in simulation. The second pilot showed that RCVM could function in a full-loop interaction system implemented with physical robots, but it left questions about what could negatively or positively affect the success of kineme communication in a complex world. Our multi-dimensional study explored four different types of communication on three different robots from a variety of viewpoints, attempting to find clarity for factors that could affect kinemes' success. Our results were extensive, but the most salient information gathered was the following: Kinemes perform remarkably similarly at all viewpoints tested, remaining much more stable in their performance than other systems, even though their overall accuracy was low. This suggests that kinemes have some level of performance invariance to viewpoint changes that other systems do not. The minimal effect of viewpoint on accuracy and operational accuracy is observed and validated through significance tests for kinemes overall. Through pairwise significance tests, we further show that each robot implementation of RCVM is minimally effected by changes in viewpoint in terms of accuracy and operational accuracy. This quality, along with the ease of learning established in Pilot I, the general viability established in Pilot II, and the varied results in the multi-dimensional study, establishes the use of RCVM for field robots as an option to be carefully considered and further explored. A great deal of work is still to be done on improving RCVM and exploring the best approaches to field HRI beyond this simple problem of creating one successful robot-to-human communication method. With the work presented in this article as a basis, we will continue our work toward the ideal of fully realized, naturally communicative robots working alongside humans in the field.

\section{ACKNOWLEDGMENTS}

The authors wish to thank the following: Noah Seichter, Hannah Dubois, Mustaf Ahmed, Kheim Vuong, Muntaqim Mehtaz, Owen Queeglay, Jungseok Hong, Karin de Langis, and Sophie Fulton for their contributions to this work, in helping to prepare or administer studies, edit and analyze video, and general advice/suggestions. Additionally, the authors thank the Minnesota Robotics Institute and the National Science Foundation for their support in our research.

\section{REFERENCES}

[1] Elena Andonova and Holly A. Taylor. 2012. Nodding in dis/agreement: A tale of two cultures. Cognitive Processing 13, 1 (2012), 79-82.

[2] Dane Archer. 1997. Unspoken diversity: Cultural differences in gestures. Qualitative Sociology 20, 1 (March 1997), 79-105. https://doi.org/10.1023/A:1024716331692

[3] Dante Arroyo, Cesar Lucho, Silvia Julissa Roncal, and Francisco Cuellar. 2014. Daedalus: A sUAV for human-robot interaction. In Proceedings of the 2014 ACM/IEEE International Conference on Human-Robot Interaction. ACM, New York, NY, 116-117. https://doi.org/10.1145/2559636.2563709

[4] Kim Baraka and Manuela M. Veloso. 2018. Mobile service robot state revealing through expressive lights: Formalism, design, and evaluation. International fournal of Social Robotics 10 (Jan. 2018), 65-92. https://doi.org/ 10.1007/s12369-017-0431-x

[5] E. Beachly, C. Detweiler, S. Elbaum, D. Twidwell, and B. Duncan. 2017. UAS-Rx interface for mission planning, fire tracking, fire ignition, and real-time updating. In Proceedings of the 2017 IEEE International Symposium on Safety, Security, and Rescue Robotics (SSRR'17). IEEE, Los Alamitos, CA, 67-74. https://doi.org/10.1109/SSRR.2017.8088142

[6] Cindy L. Bethel. 2009. Robots without Faces: Non-Verbal Social Human-Robot Interaction. PhD Dissertation. University of South Florida.

[7] C. L. Bethel and R. R. Murphy. 2008. Survey of non-facial/non-verbal affective expressions for appearance-constrained robots. IEEE Transactions on Systems, Man, and Cybernetics, Part C (Applications and Reviews) 38, 1 (2008), 83-92. https://doi.org/10.1109/TSMCC.2007.905845

[8] Cindy L. Bethel and Robin R. Murphy. 2011. Non-facial and non-verbal affective expression for appearanceconstrained robots used in victim management. Paladyn, fournal of Behavioral Robotics 1, 4 (2011), 219-230. https:// doi.org/10.2478/s13230-011-0009-5 
[9] Carlo Bonferroni. 1936. Teoria statistica delle classi e calcolo delle probabilita. Pubblicazioni del R Istituto Superiore di Scienze Economiche e Commericiali di Firenze 8 (1936), 3-62.

[10] Robin Bradbeer, S. Harrold, Francis Nickols, and Lam Fat Yeung. 1997. An underwater robot for pipe inspection. In Proceedings of the 4th Annual Conference on Mechatronics and Machine Vision in Practice. IEEE, Los Alamitos, CA, 152-156. https://doi.org/10.1109/MMVIP.1997.625313

[11] C. Breazeal. 2004. Social interactions in HRI: The robot view. IEEE Transactions on Systems, Man, and Cybernetics, Part C (Applications and Reviews) 34, 2 (2004), 181-186. https://doi.org/10.1109/TSMCC.2004.826268

[12] Cynthia Breazeal and Paul Fitzpatrick. 200. That certain look: Social amplification of animate vision. In Proceedings of the AAAI Fall Symposium on Society of Intelligence agents-The Human in the Loop. 5.

[13] John Brown. 1958. Some tests of the decay theory of immediate memory. Quarterly fournal of Experimental Psychology 10, 1 (1958), 12-21. https://doi.org/10.1080/17470215808416249 arXiv:https://doi.org/10.1080/17470215808416249

[14] Jessica Rebecca Cauchard, Kevin Y. Zhai, Marco Spadafora, and James A. Landay. 2016. Emotion encoding in human-drone interaction. In Proceedings of the 11th ACM/IEEE International Conference on Human-Robot Interaction (HRI'16). ACM, New York, NY, 263-270. https://doi.org/10.1109/HRI.2016.7451761

[15] Arturo Gomez Chavez, Andrea Ranieri, Davide Chiarella, Enrica Zereik, Anja Babic, and Andreas Birk. 2018. CADDY underwater stereo-vision dataset for human-robot interaction (HRI) in the context of diver activities. abs/1807.04856 (2018). arXiv:1807.04856 http://arxiv.org/abs/1807.04856.

[16] Joe Crumpton and Cindy L. Bethel. 2016. A survey of using vocal prosody to convey emotion in robot speech International fournal of Social Robotics 8, 2 (2016), 271-285.

[17] Kevin J. DeMarco, Michael E. West, and Ayanna M. Howard. 2014. Underwater human-robot communication: A case study with human divers. In Proceedings of the 2014 IEEE International Conference on Systems, Man, and Cybernetics (SMC'14). IEEE, Los Alamitos, CA, 3738-3743. https://doi.org/10.1109/SMC.2014.6974512

[18] Anca D. Dragan. 2015. Legible Robot Motion Planning. Ph. D. Dissertation. Carnegie Mellon University.

[19] Anca D. Dragan, Shira Bauman, Jodi Forlizzi, and Siddhartha S. Srinivasa. 2015. Effects of robot motion on humanrobot collaboration. In Proceedings of the 2015 10th ACM/IEEE International Conference on Human-Robot Interaction (HRI'15). ACM, New York, NY, 51-58.

[20] Gregory Dudek, Philippe Giguere, Chris Prahacs, Shane Saunderson, Junaed Sattar, Luz Abril Torres-Mendez, Michael Jenkin, et al. 2007. AQUA: An amphibious autonomous robot. Computer 40, 1 (Jan. 2007), 46-53. https://doi.org/ 10.1109/MC.2007.6

[21] Brittany A. Duncan, Evan Beachly, Alisha Bevins, Sebastian Elbaum, and Carrick Detweiler. 2018. Investigation of communicative flight paths for small unmanned aerial systems. In Proceedings of the 2018 IEEE International Conference on Robotics and Automation (ICRA'18). IEEE, Los Alamitos, CA, 602-609. https://doi.org/10.1109/ICRA.2018.8462871

[22] Chelsey Edge, Sadman Sakib Enan, Michael Fulton, Jungseok Hong, Jiawei Mo, Kimberly Barthelemy, Hunter Bashaw, et al. 2020. Design and experiments with LoCO AUV: A low cost open-source autonomous underwater vehicle. In Proceedings of the 2020 IIEEE/RSf International Conference on Intelligent Robots and Systems (IROS'20). IEEE, Los Alamitos, CA.

[23] Michael Fulton, Mustaf Ahmed, and Junaed Sattar. 2019. By land, air, or sea: Multi-domain robot communication via motion. CoRR abs/1903.03134 (2019). arXiv:1903.03134 http://arxiv.org/abs/1903.03134.

[24] Michael Fulton, Chelsey Edge, and Junaed Sattar. 2019. Robot communication via motion: Closing the underwater human-robot interaction loop. In Proceedings of 2019 International Conference on Robotics and Automation (ICRA'19). IEEE, Los Alamitos, CA, 4660-4666. https://doi.org/10.1109/ICRA.2019.8793491

[25] Paul A. Games and John F. Howell. 1976. Pairwise multiple comparison procedures with unequal n's and/or variances: A Monte Carlo study. Fournal of Educational Statistics 1, 2 (1976), 113-125.

[26] Shuzhi Sam Ge, Chen Wang, and Chang Chieh Hang. 2008. Facial expression imitation in human robot interaction. In Proceedings of the 17th IEEE International Symposium on Robot and Human Interactive Communication (RO-MAN'08). IEEE, Los Alamitos, CA, 213-218. https://doi.org/10.1109/ROMAN.2008.4600668

[27] Arturo Gomez Chavez, Christian Mueller, Tobias Doernbach, Davide Chiarella, and Andreas Birk. 2018. Robust gesture-based communication for underwater human-robot interaction in the context of search and rescue diver missions. In Proceedings of the IROS Workshop on Human-Aiding Robotics.

[28] John Paulin Hansen, Alexandre Alapetite, I. Scott MacKenzie, and Emilie Møllenbach. 2014. The use of gaze to control drones. In Proceedings of the Symposium on Eye Tracking Research and Applications (ETRA'14). ACM, New York, NY, 27-34. https://doi.org/10.1145/2578153.2578156

[29] Justin Hart, Reuth Mirsky, Xuesu Xiao, Stone Tejeda, Bonny Mahajan, Jamin Goo, Kathryn Baldauf, Sydney Owen, and Peter Stone. 2020. Using human-inspired signals to disambiguate navigational intentions. In Proceedings of the 12th International Conference on Social Robotics (ICSR'20). http://www.cs.utexas.edu/users/ai-lab?ICSR2020-HART.

[30] Rachel Holladay, Anca Dragan, and Siddhartha Srinivasa. 2014. Legible robot pointing. In Proceedings of the IEEE International Workshop on Robot and Human Interactive Communication. IEEE, Los Alamitos, CA. https://doi.org/ 10.1109/ROMAN.2014.6926256 
[31] Sandy H. Huang, Isabella Huang, Ravi Pandya, and Anca D. Dragan. 2019. Nonverbal robot feedback for human teachers. CoRR abs/1911.02320 (2019). arXiv:1911.02320 http://arxiv.org/abs/1911.02320.

[32] Md. Jahidul Islam, Michael Fulton, and Junaed Sattar. 2018. Toward a generic diver-following algorithm: Balancing robustness and efficiency in deep visual detection. IEEE Robotics and Automation Letters 4, 1 (2018), 113-120.

[33] Md. Jahidul Islam, Marc Ho, and Junaed Sattar. 2018. Dynamic reconfiguration of mission parameters in underwater human-robot collaboration. In Proceedings of the 2018 IEEE International Conference on Robotics and Automation (ICRA'18). IEEE, Los Alamitos, CA, 1-8. https://doi.org/10.1109/ICRA.2018.8461197

[34] Klaus Krippendorff. 2011. Agreement and information in the reliability of coding. Communication Methods and Measures 5 (April 2011), 93-112. https://doi.org/10.1080/19312458.2011.568376

[35] Henry B. Mann and D. R. Whitney. 1947. On a test of whether one of two random variables is stochastically larger than the other. Annals of Mathematical Statistics 18, 1 (1947), 50-60. http://www.jstor.org/stable/2236101

[36] Anirban Mazumdar, Martin Lozano, Aaron Fittery, and Harry Asada. 2012. A compact, maneuverable, underwater robot for direct inspection of nuclear power piping systems. In Proceedings of the IEEE International Conference on Robotics and Automation (ICRA'12). IEEE, Los Alamitos, CA, 2818-2823. https://doi.org/10.1109/ICRA.2012.6224619

[37] Mark Micire, Terrence Fong, Ted Morse, Eric Park, Chris Provencher, Ernest Smith, Vinh To, R. Jay Torres, D. W. Wheeler, and David Mittman. 2013. Smart spheres: A telerobotic free-flyer for intravehicular activities in space. In Proceedings of the AIAA Space 2013 Conference and Exposition. 5338.

[38] Valiallah Mani Monajjemi, Jens Wawerla, Richard Vaughan, and Greg Mori. 2013. HRI in the sky: Creating and commanding teams of UAVs with a vision-mediated gestural interface. In Proceedings of the IEEE/RSf International Conference on Intelligent Robots and Systems (IROS'13). IEEE, Los Alamitos, CA, 617-623. https://doi.org/ 10.1109/IROS.2013.6696415

[39] Lilia Moshkina and Ronald C. Arkin. 2005. Human perspective on affective robotic behavior: A longitudinal study. In Proceedings of the 2005 IEEE/RSf International Conference on Intelligent Robots and Systems (IROS'05). IEEE, Los Alamitos, CA, 1444-1451. https://doi.org/10.1109/IROS.2005.1545343

[40] Dula Nad, Filip Mandic, and Nikola Miskovic. 2020. Using autonomous underwater vehicles for diver tracking and navigation aiding. Fournal of Marine Science and Engineering 8, 6 (2020), 413. https://doi.org/10.3390/jmse8060413

[41] Jawad Nagi, Alessandro Giusti, Gianni A. Di Caro, and Luca M. Gambardella. 2014. Human control of UAVs using face pose estimates and hand gestures. In Proceedings of the 2014 ACM/IEEE International Conference on Human-Robot Interaction. ACM, New York, NY, 252-253. https://doi.org/10.1145/2559636.2559833

[42] Wai Shan Ng and Ehud Sharlin. 2011. Collocated interaction with flying robots. In Proceedings of the 21st IEEE International Symposium on Robot and Human Interactive Communication (RO-MAN'11). IEEE, Los Alamitos, CA, 143-149. https://doi.org/10.1109/ROMAN.2011.6005280

[43] Norman S. Nise. 2015. Control Systems Engineering (7th ed.). Wiley, NJ. http://gen.lib.rus.ec/book/index.php?md5= d89ffd2789223fd1b3d1811615d1b3ba.

[44] Winfried Noth. 1995. Handbook of Semiotics. Indiana University Press, Bloomington, IN.

[45] Cheng Peng, Pravakar Roy, James Luby, and Volkan Isler. 2016. Semantic mapping of orchards. In Proceedings of the 5th IFAC Conference on Sensing, Control, and Automation Technologies for Agriculture (AGRICONTROL'16). 85-89. https://doi.org/10.1016/j.ifacol.2016.10.016

[46] Lloyd Peterson and Margaret Jean Peterson. 1959. Short-term retention of individual verbal items. Journal of Experimental Psychology 58, 3 (1959), 193-198. https://doi.org/10.1037/h0049234

[47] Morgan Quigley, Ken Conley, Brian Gerkey, Josh Faust, Tully Foote, Jeremy Leibs, Rob Wheeler, and Andrew Y. Ng. 2009. ROS: An open-source Robot Operating System. In Proceedings of the ICRA Workshop on Open Source Software, Vol. 3. IEEE, Los Alamitos, CA, 5.

[48] J. Sattar and G. Dudek. 2009. Underwater human-robot interaction via biological motion identification. In Robotics: Science and Systems V. MIT Press, Cambridge, MA, 185-192. https://doi.org/10.15607/RSS.2009.V.024

[49] Mark Scheeff, John Pinto, Kris Rahardja, Scott Snibbe, and Robert Tow. 2002. Experiences with Sparky, a Social Robot. In Socially Intelligent Agents. Springer, Boston, MA, 173-180. https://doi.org/10.1007/0-306-47373-9_21

[50] Takuji Shimokawa and Tetsuo Sawaragi. 2001. Acquiring communicative motor acts of social robot using interactive evolutionary computation. In Proceedings of the 2001 IEEE International Conference on Systems, Man, and Cybernetics, Vol. 3. IEEE, Los Alamitos, CA, 1396-1401. https://doi.org/10.1109/ICSMC.2001.973477

[51] SoftBank Robotics EMEA. 2019. Pepper the Humanoid Robot. Softbank Robotics. https://www.softbankrobotics.com/ emea/en/pepper.

[52] Rainer Stiefelhagen, C. Fugen, R. Gieselmann, H. Holzapfel, K. Nickel, and A. Waibel. 2004. Natural human-robot interaction using speech, head pose and gestures. In Proceedings of the 2004 IEEE/RSF International Conference on Intelligent Robots and Systems (IROS'04), Vol. 3. IEEE, Los Alamitos, CA, 2422-2427. https://doi.org/10.1109/IROS.2004.1389771

[53] Daniel Szafir, Bilge Mutlu, and Terrence Fong. 2014. Communication of intent in assistive free flyers. In Proceedings of the 2014 ACM/IEEE International Conference on Human-Robot Interaction (HRI'14). ACM, New York, NY, 358-365. https://doi.org/10.1145/2559636.2559672 
[54] Daniel Szafir, Bilge Mutlu, and Terry Fong. 2015. Communicating directionality in flying robots. In Proceedings of the 10th Annual ACM/IEEE International Conference on Human-Robot Interaction (HRI'15). ACM, New York, NY, 19-26. https://doi.org/10.1145/2696454.2696475

[55] Tsuyoshi Tojo, Yosuke Matsusaka, Tomotada Ishii, and Tetsunori Kobayashi. 2000. A conversational robot utilizing facial and body expressions. In Proceedings of the IEEE International Conference on Systems, Man, and Cybernetics (SMC'00), Vol. 2. IEEE, Los Alamitos, CA, 858-863. https://doi.org/10.1109/ICSMC.2000.885957

[56] Pratap Tokekar, Joshua Vander Hook, David Mulla, and Volkan Isler. 2016. Sensor planning for a symbiotic UAV and UGV system for precision agriculture. IEEE Transactions on Robotics 32, 6 (Dec. 2016), 1498-1511. https:// doi.org/10.1109/TRO.2016.2603528

[57] Yu Ukai and Jun Rekimoto. 2013. Swimoid: Interacting with an underwater buddy robot. In Proceedings of the 8th ACM/IEEE International Conference on Human-Robot Interaction (HRI'13). ACM, New York, NY, 423-423. https:// doi.org/10.1109/HRI.2013.6483628

[58] Bart Verzijlenberg and Michael Jenkin. 2010. Swimming with robots: Human robot communication at depth. In Proceedings of the 2010 IEEE/RSf International Conference on Intelligent Robots and Systems (IROS'10). IEEE, Los Alamitos, CA, 4023-4028. https://doi.org/10.1109/IROS.2010.5652751

[59] J. Wainer, D. J. Feil-Seifer, D. A. Shell, and M. J. Mataric. 2009. The role of physical embodiment in human-robot interaction. In Proceedings of the 15th IEEE International Symposium on Robot and Human Interactive Communication (ROMAN'06). IEEE, Los Alamitos, CA, 117-122. https://doi.org/10.1109/ROMAN.2006.314404

[60] Otto Waris, Anna Soveri, Miikka Ahti, Russell Hoffing, Daniel Ventus, Susanne Jaeggi, Aaron Seitz, and Matti Laine. 2017. A latent factor analysis of working memory measures using large-scale data. Frontiers in Psychology 8 (June 2017), 1-14. https://doi.org/10.3389/fpsyg.2017.01062

[61] Stefan Williams and Ian Mahon. 2004. Simultaneous localisation and mapping on the Great Barrier Reef. In Proceedings of the 2004 IEEE International Conference on Robotics and Automation (ICRA'04), Vol. 2. IEEE, Los Alamitos, CA, 1771-1776. https://doi.org/10.1109/ROBOT.2004.1308080

[62] Melonee Wise, Michael Ferguson, Derek King, Eric Diehr, and David Dymesich. 2016. Fetch and freight: Standard platforms for service robot applications. In Proceedings of the Workshop on Autonomous Mobile Service Robots. 1-6.

[63] Allan Zhou, Dylan Hadfield-Menell, Anusha Nagabandi, and Anca D. Dragan. 2017. Expressive robot motion timing. In Proceedings of the 2017 ACM/IEEE International Conference on Human-Robot Interaction (HRI'17). ACM, New York, NY, 22-31. https://doi.org/10.1145/2909824.3020221

Received December 2020; revised July 2021; accepted October 2021 